\title{
The VIPERS Multi-Lambda Survey
}

\section{UV and near-IR observations, multi-colour catalogues, and photometric redshifts ${ }^{\star} \star \star$}

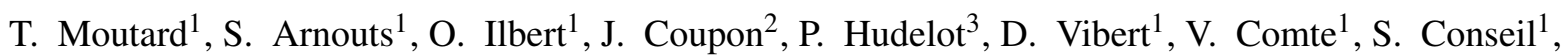 \\ I. Davidzon ${ }^{1,4}$, L. Guzzo ${ }^{5}$, A. Llebaria ${ }^{1}$, C. Martin ${ }^{6}$, H. J. McCracken ${ }^{3}$, B. Milliard ${ }^{1}$, G. Morrison ${ }^{7,8}$, \\ D. Schiminovich ${ }^{9}$, M. Treyer ${ }^{1}$, and L. Van Werbaeke ${ }^{10}$ \\ 1 Aix Marseille Université, CNRS, LAM - Laboratoire d'Astrophysique de Marseille, 38 rue F. Joliot-Curie, 13388 Marseille, \\ France \\ e-mail: thibaud.moutard@lam.fr \\ 2 Astronomical Observatory of the University of Geneva, ch. d'Ecogia 16, 1290 Versoix, Switzerland \\ 3 CNRS, UMR 7095 \& UPMC, Institut d'Astrophysique de Paris, 98bis boulevard Arago, 75014 Paris, France \\ 4 INAF-Osservatorio Astronomico di Bologna, via Ranzani 1, 40127 Bologna, Italy \\ 5 INAF-Osservatorio Astronomico di Brera, via E. Bianchi 46, 23807 Merate/via Brera 28, 20122 Milano, Italy \\ ${ }^{6}$ California Institute of Technology, Pasadena, CA 91125, USA \\ 7 Institue for Astronomy, University of Hawaii, Honolulu, HI 96822, USA \\ 8 Canada-France-Hawaii Telescope, 65-1238 Mamalahoa Highway, Kamuela, HI 96743, USA \\ 9 Department of Astronomy, Columbia University, New York, NY 10027, USA \\ 10 Department of Physics and Astronomy, University of British Columbia, 6224 Agricultural Road, Vancouver, V6T 1Z1, BC, Canada
}

Received 10 December 2015 / Accepted 9 February 2016

\begin{abstract}
We present observations collected in the CFHTLS-VIPERS region in the ultraviolet with the GALEX satellite (far- and near-ultraviolet channels) and in the near-infrared with the CFHT/WIRCam camera $\left(K_{\mathrm{s}}\right.$ band) over an area of 22 and $27 \mathrm{deg}^{2}$, respectively. The depth of the photometry was optimised to measure the physical properties (e.g., star formation rate, stellar masses) of all the galaxies in the VIPERS spectroscopic survey. The large volume explored by VIPERS will enable a unique investigation of the relationship between the galaxy properties and their environment (density field and cosmic web) at high redshift $(0.5 \leq z \leq 1.2)$. In this paper, we present the observations, the data reductions, and the build-up of the multi-colour catalogues. The CFHTLS-T0007 (gri- $\chi^{2}$ ) images are used as reference to detect and measure the $K_{\mathrm{s}}$-band photometry, while the T0007 $u^{*}$-selected sources are used as priors to perform the GALEX photometry based on a dedicated software (EMphot). Our final sample reaches $\mathrm{NUV}_{\mathrm{AB}} \sim 25$ (at $5 \sigma$ ) and $K_{\mathrm{AB}} \sim 22$ (at $3 \sigma$ ). The large spectroscopic sample ( $\sim 51000$ spectroscopic redshifts) allows us to highlight the robustness of our star/galaxy separation and the reliability of our photometric redshifts with a typical accuracy of $\sigma_{z} \leq 0.04$ and a fraction of catastrophic failures $\eta \leq 2 \%$ down to $i \sim 23$. We present various tests on the $K_{\mathrm{s}}$-band completeness and photometric redshift accuracy by comparing our results with existing overlapping deep photometric catalogues. Finally, we discuss the $B z K$ sample of passive and active galaxies at high redshift and the evolution of galaxy morphology in the (NUV $-r)$ vs. $\left(r-K_{\mathrm{s}}\right)$ diagram at low redshift $(z \leq 0.25)$ based on the high image quality of the CFHTLS.
\end{abstract}

Key words. galaxies: photometry - galaxies: distances and redshifts - galaxies: evolution

\section{Introduction}

The gain in sensitivity and resolution of modern instruments has given access to the whole electromagnetic spectrum, allowing observations of the stellar light (thermal emission from the far-ultraviolet to the mid-infrared) and of the re-processed (dust in far-infrared/radio) and non-thermal (supernovae [SN], active galactic nuclei [AGN] in radio, mid-infrared and X-ray) emissions of distant galaxies. In the past decade a wealth of

\footnotetext{
* The catalogue is available at the CDS via anonymous ftp to cdsarc.u-strasbg. fr (130.79.128.5) or via http://cdsarc.u-strasbg.fr/viz-bin/qcat?J/A+A/590/A102 $\star \star$ The images, catalogues, and photometric redshifts for 1.5 million sources (down to NUV $\leq 25 \cup K_{\mathrm{s}} \leq 22$ ) are released and available at: http://cesam.lam.fr/vipers-mls/
}

multi-wavelength data has been collected in the so-called deep fields to measure galaxy physical properties such as stellar mass, star formation activity, dust content, and AGN fraction: GOODS (Giavalisco et al. 2004), VVDS (Le Fèvre et al. 2005), COSMOS (Scoville et al. 2007), DEEP2 (Newman et al. 2013) or SXDS (Furusawa et al. 2008) among others. A clear picture has emerged for the global evolution of the star formation rate (SFR) and stellar mass densities over cosmic time (e.g., Madau \& Dickinson 2014). We observe a general decline of the star formation rate density since $z \sim 2$, a rapid build-up of the massive end of the mass function $(z \sim 1)$, accompanied by the emergence of quiescent galaxies. However, the physical processes responsible for these evolutions are still to be understood. The gradual decline of star formation (SF) activity could be due to the dwindling gas supply in galaxies, suppressing the fuel for 
star formation activity (in line with the slowing growth rate of massive dark matter halos, e.g., Bouché et al. 2010). At high redshift, hydrodynamical simulations suggest that cold streams (Katz et al. 2003) can penetrate deep into the dark matter halos and feed galaxies with fresh gas, allowing intense star formation activities and rapid formation of massive galaxies. Internal feedback processes (SN, AGN, virial shock heating; Cattaneo et al. 2006; Kereš et al. 2005) can then expel the gas and/or halt the SF activity and produce the early formation of massive quiescent galaxies. Alternatively, galaxies may also become quiescent as a result of environmental effects such as galaxy harassment (e.g., Farouki \& Shapiro 1981), ram-pressure stripping (Gunn \& Gott 1972), galaxy strangulation (Larson et al. 1980), or major merging. All these processes must be addressed in the global framework of large-scale structure formation and the complex filamentary network of the cosmic web, containing $90 \%$ of the baryons, in order to evaluate the relative contribution of environmental effects (nurture) versus internal effects (nature) in shaping the galaxy properties observed today.

To analyse the relationship between galaxy properties and their environment, the latter has been defined in different ways: i) galaxy clustering analyses provide a statistical link between the galaxies and their host dark matter haloes (Kauffmann et al. 2004), which, combined with their abundances, can constrain the dark matter halo occupation distribution (Zehavi et al. 2005); ii) the reconstruction of the local density field as measured with spectroscopic (Cucciati et al. 2006; Cooper et al. 2008; Kovač et al. 2010) or photometric (Scoville et al. 2013) redshifts allows high- and low-density contrast regions to be separated; iii) the direct measurement of pair, group, and cluster membership can be compared to isolated field galaxies (Haines et al. 2008; Wong et al. 2011; Peng et al. 2010; Kovač et al. 2014); and iv) the reconstruction of the large-scale structure network composed of filaments, nodes, walls, and voids (Aragón-Calvo et al. 2010; Sousbie 2011). It offers a new way to describe the anisotropic environment of the cosmic web. As mentioned before, the cosmic streams can feed galaxies in fresh gas, they also advect angular momentum into the disk of galaxies, which can lead to the alignment of the spine of spiral galaxies with the large-scale filaments, as predicted by tidal torques theory (Codis et al. 2015) and recently observed in the SDSS survey (Tempel et al. 2013).

All these investigations are performed extensively in the local Universe with the help of the large spectroscopic redshift surveys, SDSS, 2dF, and GAMA. At higher redshift, current surveys do not allow reconstructing the cosmic web because of either the small field of view or the low spectroscopic sampling rate. This situation has changed with the large VIMOS Public Extragalactic Redshift Survey (VIPERS; Guzzo et al. 2014) carried at ESO Very Large Telescope (VLT). With $\sim 100000$ spectroscopic redshifts over $24 \mathrm{deg}^{2}$ down to $i_{\mathrm{AB}}^{*}<22.5$ in the redshift range $0.5<z<1.2$ (Garilli et al. 2014), VIPERS probes a volume equivalent to the local $2 \mathrm{dF}$ survey, providing a unique high- $z$ sample to beat the cosmic variance $(\sim 10 \%)$ that affects current surveys ( $80 \%$ for GOODS down to $25 \%$ for COSMOS at $z \sim 0.8)$. Its spectroscopic sampling rate $(\sim 40 \%)$ ensures a sufficient sampling to measure the redshift-space distortions (de la Torre et al. 2013) and to reveal the cosmic web on a large scale.

The VIPERS spectroscopic survey benefits from the optical coverage of the CFHT Legacy Survey Wide (ugriz) in the W1 and W4 fields. To derive reliable stellar masses and SFR for VIPERS galaxies, we have undertaken a complete near-infrared (NIR) follow-up in the two fields and partial follow-up in near-ultraviolet (only W1), which are described in this paper. In the past ten years, stellar population synthesis (SPS) models have been extensively used to convert luminosity into physical properties using a template-fitting procedure (e.g., see recent reviews by Walcher et al. 2011; Conroy 2013). The wavelength coverage is critical to reduce the uncertainties on those parameters, however. The NIR domain samples the rest-frame optical part of the spectral energy distribution (SED) of distant galaxies. Without NIR, stellar mass measurements are uncertain $\left(\sigma_{\mathrm{M}} \sim 0.4 \mathrm{dex}\right)$, with strong systematics at $z>0.8$ that are caused by the degeneracy between age, metallicity, and extinction (Ilbert, private communication). The vast majority of VIPERS galaxies are below $z<1.0$, and the CFHTLS imaging does not fully probe the restframe ultraviolet (UV) light emitted by massive stars, which is crucial for an SFR estimate. The VIPERS area has been partially observed with the GALEX survey (Martin et al. 2005) in the farUV (135-175 nm) and near-UV (170-275 nm). However, measuring the GALEX photometry is a complex problem because of the large point-spread function of the telescope $\left(F W H M \sim 5^{\prime \prime}\right)$. The standard SExtractor photometry (Bertin \& Arnouts 1996) delivered by the GALEX pipeline is not optimal for faint sources because of blending problems. It requires a dedicated software (Conseil et al. 2011), as discussed in this paper.

With the physical properties of the VIPERS spectroscopic sources derived from the multi-wavelength dataset described in this paper, Davidzon et al. (2013) measured the high-mass tail of the galaxy stellar mass function (GSMF) with unprecedented accuracy for both quiescent and star-forming galaxies from $z=1.3$ to $z=0.5$ using the first 53608 spectroscopic redshifts from VIPERS (over $7.5 \mathrm{deg}^{2}$ ). Davidzon et al. (2016) extended their GSMF analysis as a function of environment by splitting galaxies into high- and low-density regions (using a local 3D galaxy density measurement) and detected an evolution of the GSMF in high-density regions. In contrast, the GSMF remains nearly constant in low-density regions.

In addition to the VIPERS spectroscopic sample, the photometric multi-wavelength survey $(F / \mathrm{NUV} \sim 25$, ugriz $\sim 24.5$, $\left.K_{\mathrm{s}} \sim 22\right)$ combines depth and area $\left(\sim 24 \mathrm{deg}^{2}\right)$ and provides accurate photometric redshifts and stellar masses for almost one million galaxies below $z \leq 1.5$ as well as an efficient separation between active and passive galaxies based on a NUVrK colour diagram (Arnouts et al. 2013). Moutard et al. (2016) exploited this large volume to follow the evolution of the GSMF over the past $10 \mathrm{Gyr}$ for massive galaxies, $M \geq 10^{10} M_{\odot}$. It extended the precursor work by Matsuoka \& Kawara (2010) performed in a $55 \mathrm{deg}^{2}$ region of the Stripe 82 overlapping the UKIDSSLAS $K$-band (Lawrence et al. 2007), where they constrained the growth history of the most massive galaxies $(\log (M / M o)>11$ since $z \sim 0.9$. Coupon et al. (2015) used the superb image quality of the CFHT images to perform a combined analysis of galaxy clustering, galaxy-galaxy lensing ${ }^{1}$, and the GSMF to produce a new constraint on the relationship between galaxies and their host dark matter halos.

This paper is organised as follows: we present the WIRCam- $K_{\mathrm{s}}$ and GALEX-UV observations, the data reduction and the creation of the multi-colour catalogue based on the CFHTLS optical catalogue from the final TERAPIX release (T0007). We perform various tests on our photometry and completeness by comparing them with deeper overlapping datasets and simulations. We use the large spectroscopic sample to quantify the quality of our photometric redshifts. As direct applications, we present the selection of a bright $B z K$ sample, and we

The galaxy shape is obtained with the CFHTLenS catalogues. 
illustrate the morphology of low- $z(z \leq 0.25)$ large galaxies in the $(\mathrm{NUV}-r)$ vs. $(r-K)$ rest-frame colour diagram. Throughout the paper we adopt the following cosmology: $H_{0}=70 \mathrm{~km} \mathrm{~s}^{-1} \mathrm{Mpc}^{-1}$ and $\Omega_{\mathrm{M}}=0.3, \Omega_{\Lambda}=0.7$. We adopt the initial mass function of Chabrier (2003), truncated at 0.1 and $100 M_{\odot}$. All magnitudes are given in the AB system (Oke 1974).

\section{Data}

\subsection{Optical CFHTLS photometry}

The Canada-France-Hawaii Telescope Legacy Survey ${ }^{2}$ (CFHTLS) is an imaging survey performed with MegaCam (Boulade et al. 2000) in five optical bands $u^{\star}, g, r, i, z$. The CFHTLS-Wide covers four independent patches in the sky over a total area of $154 \mathrm{deg}^{2}$ with sub-arcsecond seeing (median $\sim 0.7^{\prime \prime}$ ) and a typical depth of $i \sim 24.8$ ( $5 \sigma$ detection in $2^{\prime \prime}$ apertures). In this study we use the images and photometric catalogues of the W1 and W4 fields from the worldwide T0007 ${ }^{3}$ release produced by TERAPIX ${ }^{4}$. This final release includes an improved absolute and internal photometric calibration based on the photometric calibration scheme adopted by the Supernova Legacy Survey (SNLS) and described in Regnault et al. (2009). The main ingredients are i) the use of the spectrophotometric standard star BD +17 4708 instead of the Landolt standard stars; ii) a new photometric flat-fields used in the reduction pipeline by Elixir at CFHT on the raw images, which guarantees a flat photometry across the MegaCam field of view; and iii) the use of shallow photometric calibration observations covering the whole CFHTLS wide in each filter and bracketed by SNLS tertiary standards. These steps bring the absolute and relative photometric calibrations over the entire survey to an accuracy level of between 1 to $2 \%$.

The final images were stacked with the software SWARP (Bertin 2006). The photometry was performed with SExtractor (Bertin \& Arnouts 1996) in dual mode with the source detection based on the $g r i-\chi^{2}$ image (Szalay et al. 1999). Following Erben et al. (2013), we used the isophotal apertures, extracted from the detection images, to measure the galaxy colours. The apertures are smaller than the Kron-like apertures (Kron 1980), which provides less noisy estimates of the colours and leads to an improved photometric redshift accuracy (Hildebrandt et al. 2012).

To derive the total magnitudes, we rescaled the isophotal magnitudes (MAG_ISO) to the quasi-total magnitudes (MAG_AUTO) for each source. The scaling factor, $\delta_{m}$, combines the individual scalings in $g^{\prime}, r^{\prime}, i^{\prime}$, and $K_{\mathrm{s}}$ band, when available (the $K_{\mathrm{s}}$-band images share the same pixel grid as the optical images, see Sect. 2.2), to account for the intrinsic colours of galaxies and their respective uncertainties. It is defined as

$\delta_{m}=\frac{\sum_{f}\left(m_{\mathrm{AUTO}}-m_{\mathrm{ISO}}\right)^{f} \times w^{f}}{\sum_{f} w^{f}}$,

where $f$ corresponds to the $g^{\prime}, r^{\prime}, i^{\prime}$, and $K_{\mathrm{s}}$ filters, and the weight, $w^{f}$, is defined as $1 / w^{f}=\left(\sigma_{\text {AUTO }}^{2}+\sigma_{\text {ISO }}^{2}\right)^{f}$. The final magnitudes for each source are then simply defined by a unique shift applied to all passbands: $m^{f}=m_{\mathrm{ISO}}^{f}+\delta_{m}$.

Finally, we also investigated the best masking procedures of the bad regions by comparing the masks produced by TERAPIX

\footnotetext{
http://www.cfht.hawaii.edu/Science/CFHTLS/

http://terapix.iap.fr/cplt/T0007/doc/T0007-doc.html

4 http://terapix.iap.fr/
}
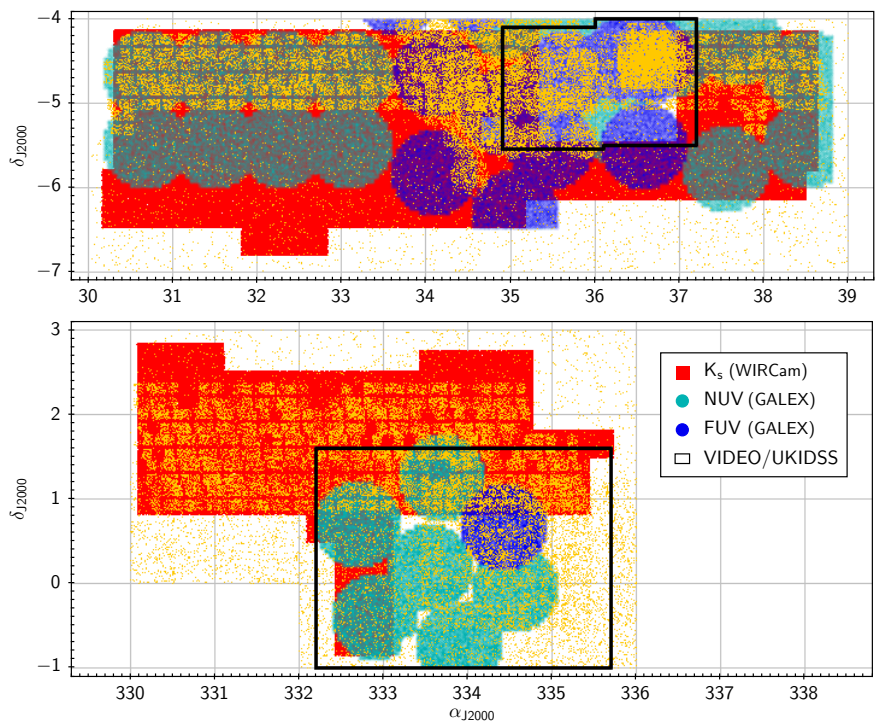

Fig. 1. Footprints of the GALEX NUV/FUV (cyan/blue) and WIRCam $K$ band (red background) observations in the CFHTLS W1 (top) and W4 (bottom) fields. The spectroscopic sources are shown as golden points. The total area (W1 and W4) is $\sim 7.8$ and $\sim 22 \mathrm{deg}^{2}$ in FUV and NUV bands observed with GALEX and $\sim 27 \mathrm{deg}^{2}$ with WIRCam. The black layouts represent the VIDEO (W1) and UKIDSS (W4) surveys.

and the CFHTLenS team ${ }^{5}$. After visual inspections, we found that CFHTLenS masks provide a better cleaning of spurious objects around bright stars by adopting a large central disk region on top of the elongated spikes (Erben et al. 2013). We therefore decided to adopt their more conservative masking approach.

In conclusion, our CFHTLS optical catalogue is similar to the original T0007 in terms of source detections, but differs in the masking regions and the choice of magnitudes, where we adopted a scheme that allows optimizing the colour estimates for the photometric redshifts and the total flux for the measurements of the physical parameters. In Fig. 1 UV (GALEX) and NIR (WIRCam $K_{\mathrm{s}}$-band) observations overlap the CFHTLS.

\subsection{NIR observations}

\subsubsection{Observations}

Since 2010, we have conducted a $K_{\mathrm{s}}$-band follow-up of the VIPERS fields with the WIRCam instrument (Puget et al. 2004) at CFHT. The original motivation was to guarantee an almost complete detection in $K_{\mathrm{s}}$ band of the VIPERS spectroscopic galaxies $\left(i_{\mathrm{AB}} \leq 22.5\right)$. To do so, we estimate that we need to detect sources down to $K_{\mathrm{s}} \sim 22$ (at $\sim 2-3 \sigma$ ), which can be reached with a minimum integration time per pixel of $\sim 1050 \mathrm{~s}$. The observations have been executed with two separated observing groups with 21 exposures of $25 \mathrm{~s}$ each. The WIRCam observations are shown in red in Fig. 1. They cover a total area of $27 \mathrm{deg}^{2}$ and correspond to a total amount of allocated time of $\sim 120 \mathrm{~h}$ spread during the period 2010 and 2012. As a result of high sky background or bad seeing $\left(I Q>0.8^{\prime \prime}\right)$, a few observing groups have been repeated. The average seeing for each WIRCam tile is shown in Fig. 2 for the two fields. The mean image quality is very homogeneous and corresponds to $\langle I Q\rangle=0.6^{\prime \prime} \pm 0.09$. We note that the missing region in the $\mathrm{W} 1$ field corresponds to the location of the VIDEO survey. The southern extension of W4 outside the VIPERS regions was planned to cover the GALEX

\footnotetext{
http://www.cfhtlens.org/
} 
field that was initially not covered by the earliest release of the UKIDSS DXS survey.

\subsubsection{Data reduction}

All the data were reduced at CFHT and TERAPIX. The initial processing with the $i$ ' wii pipeline ${ }^{6}$ at CFHT removes the instrumental imprints on the individual images (flagging of saturated, bad, and hot pixels, correcting for non-linearity, bias removal, dome and sky flat-fielding, and guide window masking). CFHT delivers detrended images with a first astrometric solution, photometric zeropoint, and a sky subtraction for each exposure.

TERAPIX first uses QualityFITS on detrended images to produce weight-maps (with the WeightWatcher software; Marmo \& Bertin 2008), source catalogues and checks the individual image qualities (e.g., seeing and depth). The validated exposures are then median-combined to produce an initial stack by using the Scamp and Swarp softwares ${ }^{7}$ (Bertin 2006). To improve the sky subtraction and the photometry of faint sources, a two-step strategy is adopted similar to the CFHT-WIRDS (Bielby et al. 2012) and the ESO-UltraVISTA (McCracken et al. 2012) surveys. The initial stack is used to produce a mask with all the faint sources. In a second pass, the mask is applied to the individual exposures and a new sky background is estimated by medianing images inside a sliding window and for images within a time window $\Delta t<15 \mathrm{~min}$ and angular separation $\Delta \theta<10^{\prime}$. A last quality assessment of the individual sky-subtracted images is performed before producing the final stacks. The final WIRCam products are composed of stack images and weight images resampled on the footprint of the CFHTLS-MegaCam tiles and with the same pixel scale $\left(0.18^{\prime \prime} /\right.$ pix $)$ using the SWARP software with a Lanczos3 resampling kernel. Like for the T0007 release, the photometry is performed in dual-image mode with the gri $-\chi^{2}$ image and the same SExtractor setting (Hudelot et al. 2012). A last correction is applied to the SExtractor error measurements, which are underestimated as a result of the noise correlation introduced by the stacking or resampling process. To estimate this factor, we also performed stacks on the native WIRCam grid (and original pixel-scale) with a nearestneighbour technique to preserve the white-noise nature in the final images. When we compared the $K_{\mathrm{s}}$ uncertainties of the same sources, we found that a factor 1.5 needed to be applied to the error estimates with the megaCam grid. No masking other than the CFHTLenS masks needed to be applied to the WIRCam sample. The effective WIRCam $K_{\mathrm{s}}$ area, after applying the masks, drops from $27 \mathrm{deg}^{2}$ to $22.4 \mathrm{deg}^{2}$ (including W1 and W4 fields). The WIRCam number counts in the two fields are shown in Fig. 3 separately for stars and galaxies (see Sect. 3) and are compared with literature.

\subsubsection{Comparison with deeper datasets}

As shown in Fig. 2, we can use the existing deep NIR surveys, UKIDSS-Ultra Deep Survey (Lawrence et al. 2007, UDS) and VIDEO (Jarvis et al. 2013), to estimate the photometric accuracy, depth, and completeness of our $K_{\mathrm{s}}$ sample. UDS and VIDEO are significantly deeper than our dataset $(K \sim 24.5$ and 23.8 respectively). For both fields the relative astrometric accuracy with our sample is $\sigma \leq 0.09^{\prime \prime}$ with systematic shifts of the same order. We adopted a maximal matching distance of $0.5^{\prime \prime}$.

\footnotetext{
6 http://cfht.hawaii.edu/Instruments/Imaging/WIRCam/

IiwiVersion1Doc.html

7 http://www.astromatic.net
}
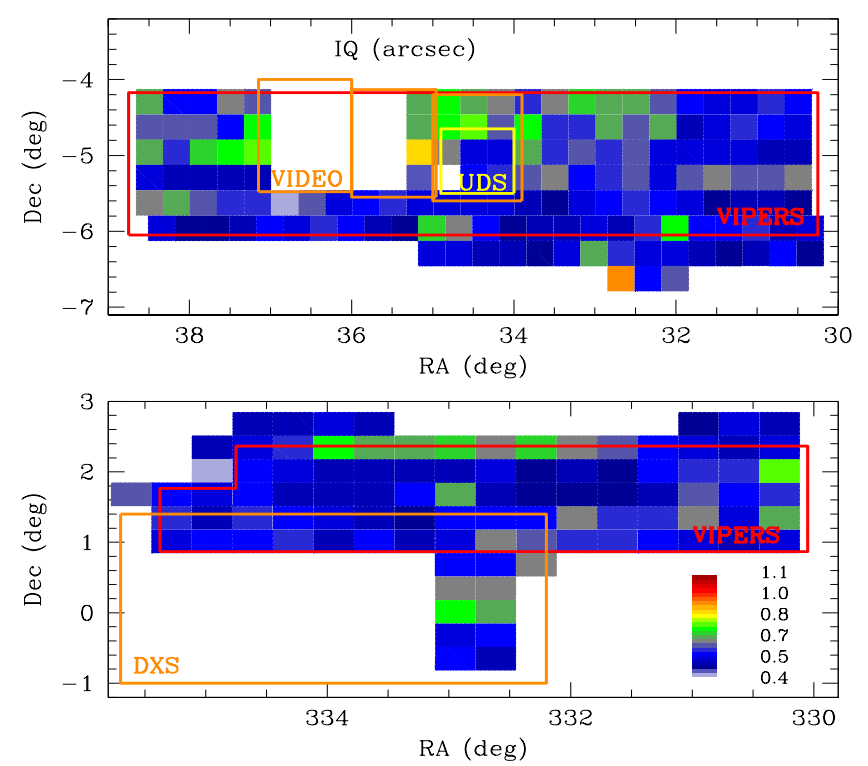

Fig. 2. Colour-coded mean image quality (IQ) per WIRCam tile over the whole WIRCam survey (W1: top and W4: bottom). The layouts indicate the VIPERS regions (red layouts), the three VIDEO pointings (orange in W1), the UKIDSS-UDS (yellow in W1), and UKIDSS-DXS (orange in W4).

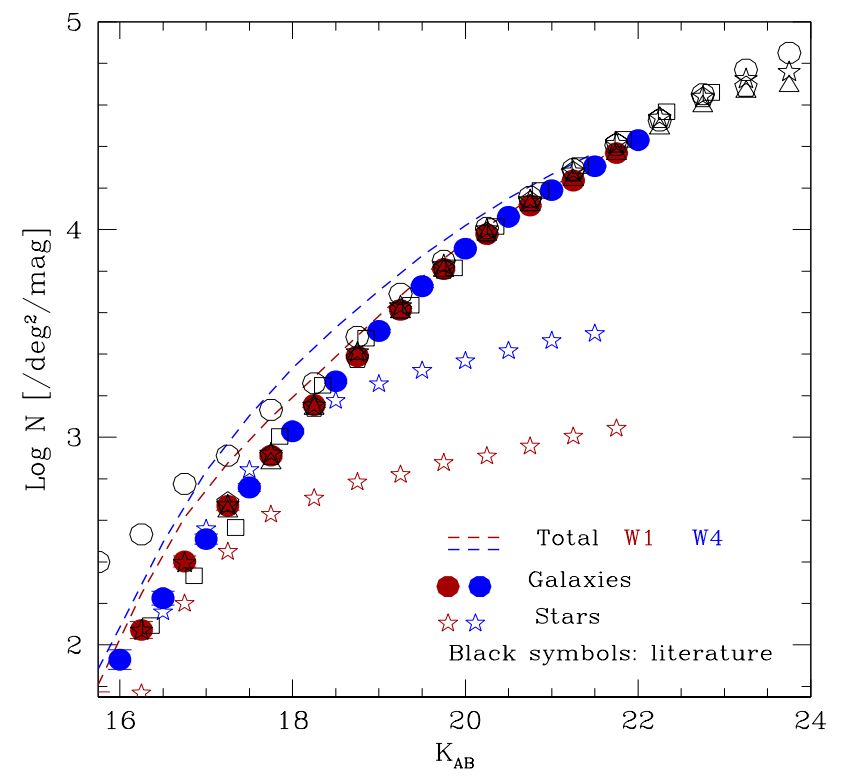

Fig. 3. WIRCam $K_{\mathrm{s}}$ number counts for the galaxies (filled circles) and stars (open stars) in the W1 (red) and W4 (blue) regions. The dashed lines show the total number counts. The open dark symbols are a compilation from the literature (triangles: McCracken et al. 2012; pentagons: Jarvis et al. 2013; circles: UDS (DR8); squares: Quadri et al. 2007; stars: Bielby et al. 2012).

To compare the fluxes, we used the SExtractor MAG_AUTO magnitudes for the VIDEO survey and the petrosian magnitudes for the UDS survey (which should be close to our own magnitudes). Figure 4 shows the magnitude differences as a function of magnitude (on the left) and the dispersions in three magnitude intervals (on the right) after correcting for the systematic shifts $\left(K_{\text {DEEP }}-K_{\text {WIRCam }} \sim 0.06\right.$ and 0.10 mag for UDS and VIDEO, respectively). For both surveys the mean difference is stable and close to zero down to $K_{\mathrm{s}} \sim 22$, confirming that no bias due to sky-background subtraction is present in our 


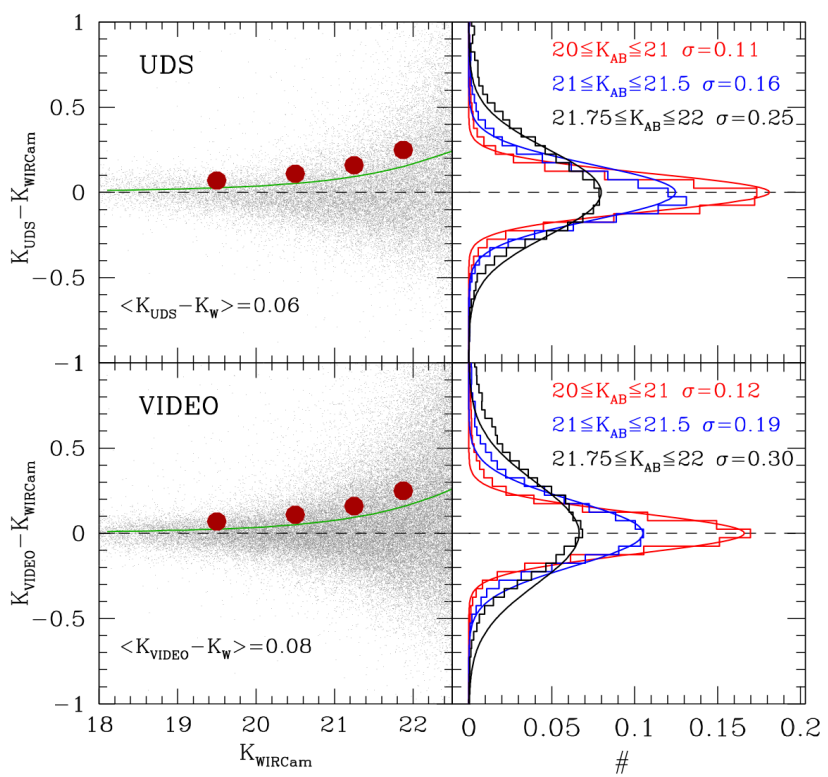

Fig. 4. Comparison of the VIPERS $K$-band photometry with the UltraDeep UKIDSS field (top left), the VIDEO XMM2 field (bottom left), and the scatter distributions in three magnitude bins (right panels). The observed scatters are reported in the left panels as red circles, while the green solid lines show the mean magnitude vs. error behaviour of our VIPERS $K$ band data after rescaling the original errors by 1.5 (see text). Larger dispersions are observed with VIDEO than with UDS because of the different depths between the two fields.

data. At all magnitudes, the dispersions are reasonably well fitted by a Gaussian distribution. We expect to reach $\sigma \sim 0.3 \mathrm{mag}$ $(S / N \sim 3)$ near $K_{\mathrm{s}} \sim 22$. The dispersions predicted with these external dataset are consistent with those estimated by SExtractor (after the 1.5 rescaling factor mentioned above).

Because we used the gri $-\chi^{2}$ images for the detection, our completeness is not directly related to the background noise of the WIRCam images. To estimate the completeness, we used all the sources detected in the UDS and VIDEO images and measured the fraction of them detected in our optical-based catalogue. In Fig. 5 (top panel) we show the completeness for the UDS (circles) and the VIDEO (squares) surveys as a function of magnitude. The solid lines and filled points refer to all the WIRCam sources irrespective of their signal-to-noise ratio $(\mathrm{S} / \mathrm{N})$. Identical results are obtained in both fields, which shows that our catalogue detects more than $85 \%$ of the pure $K_{\mathrm{s}}$-band selected surveys down to $K_{\mathrm{s}} \sim 22$. If we restrict the detection to WIRCam sources with $S / N>3$ and 5, the completeness drops slightly to $81 \%$ and $66 \%$ (based on the UDS survey), respectively, at $K_{\mathrm{AB}} \sim 22$. This confirms that our gri-based catalogue is almost complete down to the $K_{\mathrm{AB}} \sim 22 \mathrm{mag}$.

We can investigate the nature of this incompleteness in more detail, which should preferentially reflect a bias against the reddest sources. To do so, we measured the completeness as a function of $K$ magnitude and $(z-K)$ colour for the VIDEO survey alone (which includes its own $z$-band photometry that reaches a depth $z \sim 25.7$ at $5 \sigma$ ). The bidimensional completeness is shown in Fig. 5 (bottom panel). The colour-coding reflects the completeness level, with yellow corresponding to a $50 \%$ completeness. Below $K_{\mathrm{AB}} \sim 20.6$, the catalogue is complete regardless of $(z-K)$ colour. At fainter $K$ sources, the completeness of the reddest sources decreases gradually. For this reason, any statistical analysis such as luminosity functions or stellar mass
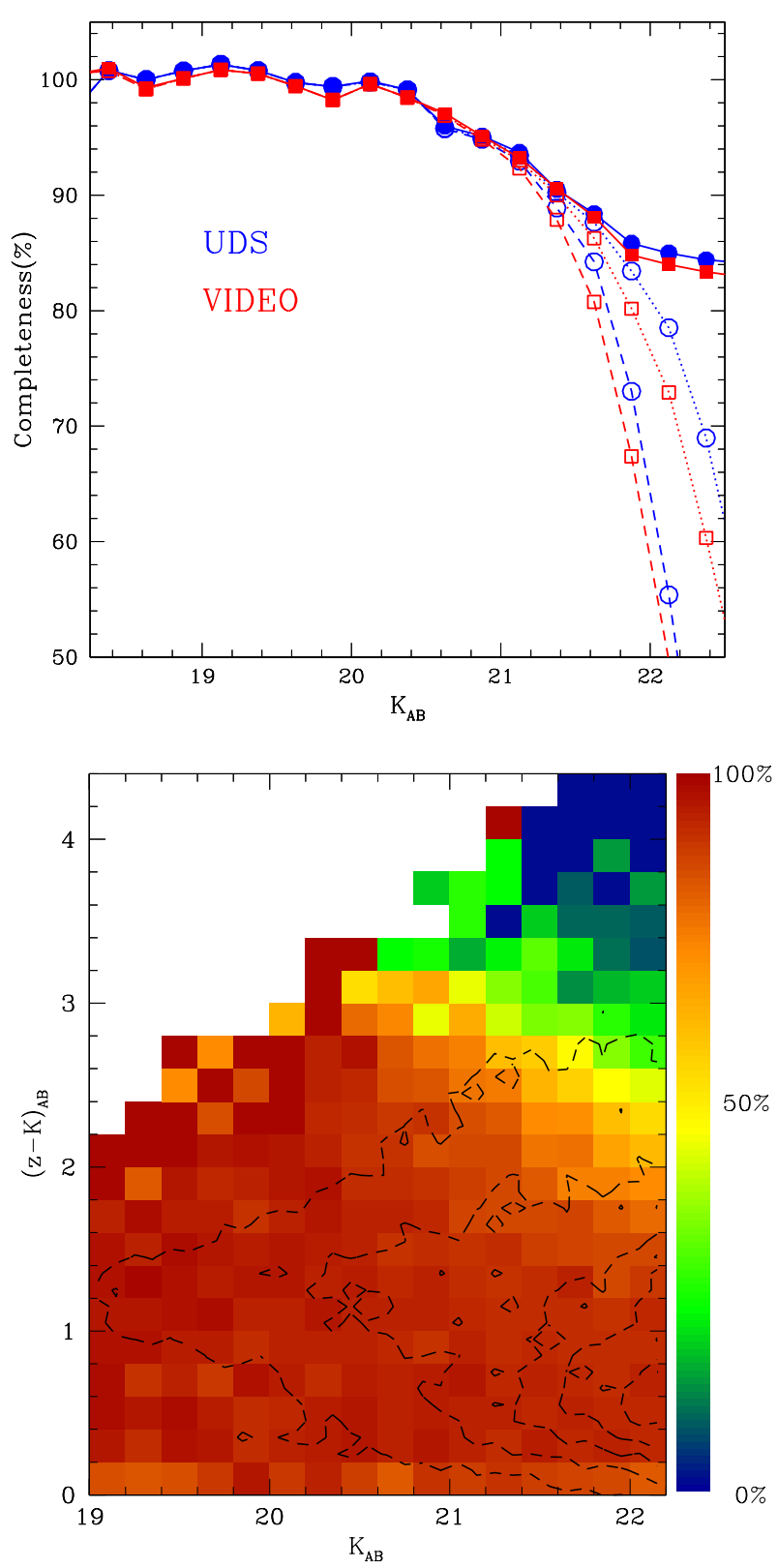

Fig. 5. Top panel: completeness (in \%) of the WIRCam sources as a function of magnitude, based on the deep surveys UDS (blue) and VIDEO (red). The completeness is shown for WIRCam sources with various signal-to-noise ratio cuts (no cut: filled symbols; $S / N>3$ : dotted lines; $S / N>5$ : dashed lines). Bottom panel: completeness as a function of magnitude and colour $(z-K)$, colour-coded from red (100\%) to blue $(0 \%)$ based on the VIDEO survey. The yellow region delineates the $50 \%$ completeness level. The dashed line contours show the source density spaced with logarithmic scale of 0.3 dex. At $K_{\mathrm{AB}} \leq 20.6$, the WIRCam sample is close to $100 \%$ completeness and the colour variations are due to small numbers.

functions, based on a $K$ selection, should apply a weight reflecting this bidimensional completeness map.

\subsection{UV photometry}

\subsubsection{Observations}

Launched in 2005, the GALEX satellite (Martin et al. 2005) has explored the ultraviolet sky in FUV (135-175 nm) and NUV 
Table 1. GALEX fields with integration times and central positions.

\begin{tabular}{|c|c|c|c|c|}
\hline \multirow[t]{2}{*}{ GALEX field } & \multicolumn{2}{|c|}{$T_{\exp }(\mathrm{ks})$} & \multirow{2}{*}{$\begin{array}{l}\text { RA } \\
(\mathrm{deg})\end{array}$} & \multirow{2}{*}{$\begin{array}{l}\text { Dec } \\
(\mathrm{deg})\end{array}$} \\
\hline & NUV & FUV & & \\
\hline \multicolumn{5}{|c|}{ W1 field } \\
\hline CFHTLS_W1_MOS00 & 27 & - & 38.5 & -4.65 \\
\hline CFHTLS_W1_MOS01 & 29 & - & 38.5 & -5.5 \\
\hline CFHTLS_W1_MOS02 & 30 & - & 7.7 & -4.28 \\
\hline CFHTLS_W1_MOS03 & 29 & - & 7.55 & -5.78 \\
\hline CFHTLS_W1_MOS04 & 28 & - & 33.1 & -4.65 \\
\hline CFHTLS_W1_MOS05 & 29 & - & 221 & -5.5 \\
\hline S_W1_MOS06 & 29 & - & & -4.65 \\
\hline CFHTLS_W1_MOS07 & 26 & _- & 2.3 & -5.5 \\
\hline CFHTLS_W1_MOS08 & 30 & - & 5 & -4.65 \\
\hline S_W1_MOS09 & 30 & - & 1.5 & -5.5 \\
\hline & 22 & - & & -4.65 \\
\hline 1 & $J$ & - & & -5.5 \\
\hline$-\bar{T} \mathrm{C}^{2}$ & 23 & - & & -3.96 \\
\hline PS_C & 3 & _- & 5.87 & -4.25 \\
\hline S_MOS04 & 35 & - & 36.9 & -4.42 \\
\hline & & - & & -5.05 \\
\hline PS & $3<+3>2$ & - & & -5.2 \\
\hline & 75 & 60 & & -4.48 \\
\hline & 115 & 92 & 36.36 & -4.48 \\
\hline $\mathrm{XM}$ & 27 & 27 & 36.58 & -5.51 \\
\hline & & 00 & 17 & -5.51 \\
\hline $\mathrm{XI}$ & 29 & 27 & 34.91 & -5.1 \\
\hline & & 26 & 36.48 & -3.60 \\
\hline & 2 & 29 & 34.6 & -3.65 \\
\hline 07 & 28 & 27 & 34.1 & -5.82 \\
\hline & 0 & 31 & 35.05 & -6.29 \\
\hline $\mathrm{XI}$ & 32 & 31 & 34.11 & -4.64 \\
\hline & 0 & 21 & & -3.68 \\
\hline XMMLSS_20 & 27 & 26 & 35.65 & -4.65 \\
\hline \multicolumn{5}{|c|}{ W4 fiel } \\
\hline VVDS & 79 & 76 & 334.4 & 0.67 \\
\hline PS_VVDS2 & 54 & - & 333.7 & 1.25 \\
\hline & 53 & - & 332.7 & 0.7 \\
\hline PS_VVDS $22 \mathrm{H} \_M O S 02$ & 59 & _- & 334.4 & 0.67 \\
\hline PS_VVDS $22 \mathrm{H}+M O S 03$ & 38 & - & 333.5 & 0.18 \\
\hline PS_VVDS $22 \mathrm{H}+M O S 04$ & 37 & - & 334.5 & -0.05 \\
\hline PS_VVDS22H_MOS05 & 52 & - & 333.9 & -0.72 \\
\hline PS VVDS22H_MOS06 & 52 & _- & 332.9 & -0.4 \\
\hline
\end{tabular}

(170-275 nm) passbands (Morrissey et al. 2005) and performed all-sky medium- and deep-imaging surveys. The UV continuum is a direct indicator (except for the dust) of the instantaneous SFR. To assess the continuum of high redshift galaxies, we here only considered the observations from the Deep Imaging Survey (DIS, with $T_{\exp } \geq 30 \mathrm{ks}$ ). All the GALEX pointings are shown in Fig. 1 as blue (FUV) and cyan (NUV) circles. To complete the coverage of the VIPERS-W1 field in the NUV channel (after the shut-off of the FUV channel), we observed new pointings in the western and eastern parts of the field with $\sim 100 \mathrm{~h}$ of discretionary time. Only the GALEX PanStarr fields, centred on the VVDS-22hr region, are available in the W4 field, with a small overlap with the VIPERS-W4 area. All the GALEX pointings and their respective integration times are given in Table 1. The total area observed with GALEX (W1 and W4) is $\sim 7.8$ and $\sim 22 \mathrm{deg}^{2}$ in the FUV and NUV bands.

\subsubsection{UV photometric extraction}

The data were reduced with the standard GALEX pipeline. In the specific case of the DIS observations, the large PSF $\left(F W H M \sim 5^{\prime \prime}\right)$ affects the extraction and photometry of faint UV sources because sources are confused (Xu et al. 2005). As an alternative to the source extraction of the GALEX pipeline (based on SExtractor software), we developed a dedicated code to perform the UV photometry, EMphot (Guillaume et al. 2005; Conseil et al. 2011). EMphot uses a Bayesian approach with optical priors based on the CFHTLS (T0007) $u^{*}$-selected sources. A full description of the code and extensive simulations will be given in a forthcoming paper; we outline the main steps below.

We generate a simulated GALEX image combining all the optical priors with a relative flux contribution that must be estimated. The priors can either be considered as a simple Dirac function or as an extended 2D profile extracted from the $u^{*}$-band image (i.e., stamps), which is what we adopted here. The formalism is the following: each observed value of the GALEX pixel $\left(x_{i}\right)$ is considered as a sample of a random variable $X_{i}$ with the expected value being $\mu_{i}=E\left\{X_{i}\right\}$. The value $\mu_{i}$ at pixel $x_{i}$ is the contribution of all the input optical priors around it and the sky background. It can be defined as

$\mu_{i}=\sum_{k=1}^{K} \alpha_{k} h_{k, i}+b_{i}$,

where $b_{i}$ is the background level value, $h_{k, i}=\sum_{j} o_{k, j} f_{i-j}$ is the sum of all the objects, $o_{k}$, convolved by the GALEX PSF, $f$. The function $h_{k, i}$ is normalised to unity and a set of scaling factor, $\alpha_{k}$, is applied to each prior $k$.

Here, we considered that the variable $X_{i}$ follows a Poisson statistic: $P\left\{X_{i}=x_{i}\right\}=\exp \left(-\mu_{i}\right) \frac{\mu_{i}^{x_{i}}}{x_{i} !}$.

To estimate the $\alpha_{k}$ values, Guillaume et al. (2005) introduced the expectation maximisation scheme (EM, see Horiuchi et al. 2001), which leads to the iterative process

$\alpha_{k}^{(n+1)}=\alpha_{k}^{(n)} \frac{\sum_{i=1}^{M}\left(x_{i} / \mu_{i}^{(n)}\right) h_{k, i}}{\sum_{i=1}^{M} h_{k, i}}$,

where $\mu_{i}^{(n)}=\sum_{j=1}^{K} \alpha_{j}^{(n)} h_{j, i}+b_{i}$. The E step compares the data image $x_{i}$ to the projection $\mu_{i}^{(n)}$ of the $\alpha_{i}^{(n)}$ estimates. The result is introduced in the $M$ step as the corrective ratio needed for the new set of $\alpha_{k}^{(n+1)}$ estimates. Although the global system may take very many iterations to converge, we found that 100 iterations is sufficient to obtain stable $\alpha_{k}$ values for the main contributors, while priors with negligible values may oscillate. To estimate the error of object $k$, we empirically quantify the variance $\sigma_{k}$, by the rms under the object's area of the residual (best-fit model subtracted from the observed image) as

$\sigma_{k}^{2}=\frac{\sum_{i=1}^{M} h_{k, i}\left(x_{i}-\hat{\mu}_{i}\right)^{2}}{\sum_{i=1}^{M} h_{k, i}^{2}}$.

We find that this estimate is consistent with the errors obtained from simulations.

We tested the EM photometric technique by adding multiple sets of simulated sources inside the observed GALEX images. In Fig. 6 we show the flux ratio between the EM fluxes and the simulated fluxes as a function of magnitude. The EM algorithm allows us to derive accurate UV fluxes down to NUV $\sim 25$ with a $S / N \geq 5$. This is consistent with the theoretical estimate of the $\mathrm{S} / \mathrm{N}$ inside the PSF aperture and the measured background 


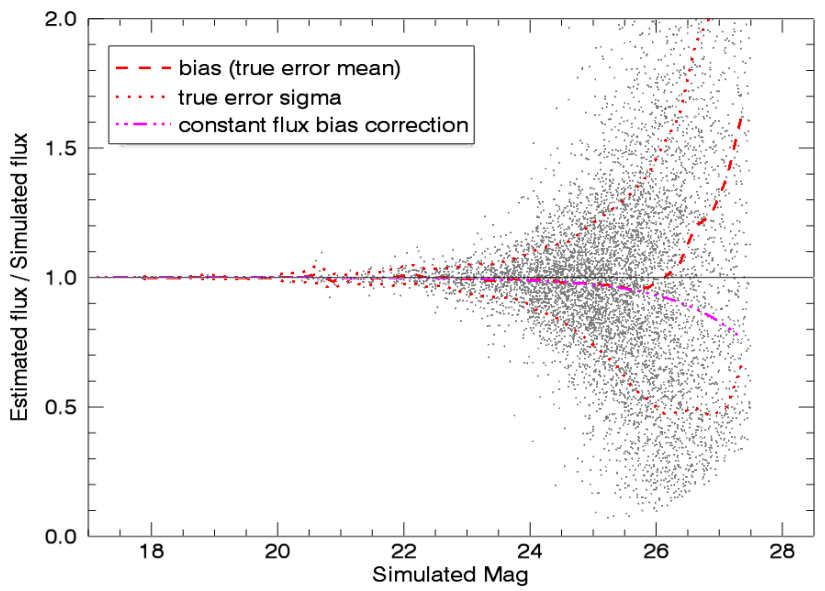

Fig. 6. Comparison of the EM vs. simulated fluxes as a function of simulated magnitudes. The dotted lines show the $1 \sigma$ error, the red dashed line the mean bias. The magenta dot-dashed line reflects the effect of a small background bias on the flux ratio.

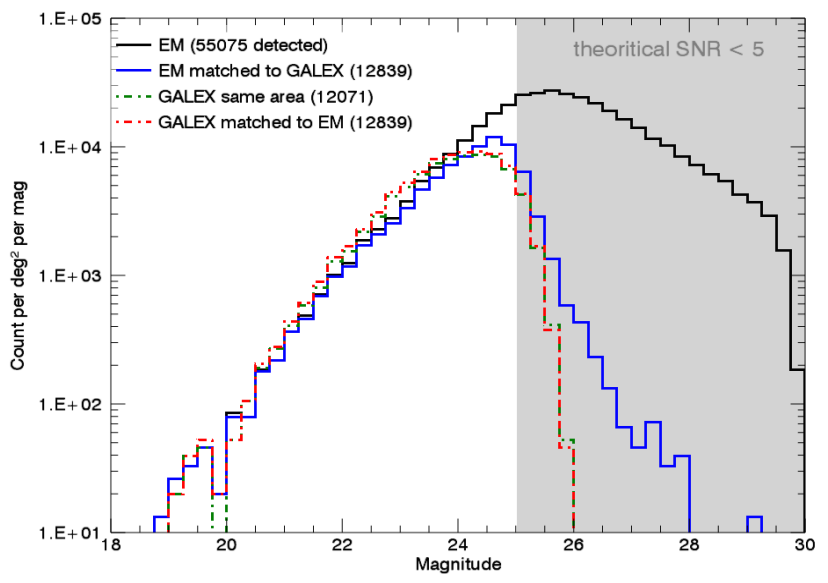

Fig. 7. NUV number counts vs. magnitude in one GALEX field. The full EM photometric catalogue is shown as a solid black line and the EM sources matched to the GALEX pipeline sources as a solid blue line. The GALEX pipeline counts in the same area (and matched to EM sources) are shown as red and green dot-dashed lines.

level. We observe a negligible mean bias (red dashed line) down to NUV 26, and its behaviour can be reproduced by a small residual background offset (magenta dot-dashed line).

In Fig. 7 we show the NUV number counts from the EM software (black line) and the GALEX pipeline (green line) in one GALEX field. First, the GALEX pipeline shows an excess of bright sources $(22 \leq \mathrm{NUV} \leq 25)$. This is explained by the deblending of the neighbouring objects in the EM technique, which leads to systematically lower fluxes for EM. This is illustrated by comparing the number counts for the same objects as a function of GALEX (red line) and EM (blue line) magnitudes. Second, the GALEX number count drops shortly after NUV $\sim 24$ (red line), while EM counts continue to increase down to NUV $\sim 25$. This is indeed expected because we used optical priors and because the sources were deblended.

To conclude, the EMphot algorithm allows us to address the problem that is related to the GALEX confusion. It provides a significative improvement with respect to the GALEX pipeline photometry for the deep fields and allows us to make full use of the GALEX photometry down to the nominal S/N of the observations. The GALEX number counts with EM photometry in the FUV and NUV channels are shown in Fig. 8 separately for

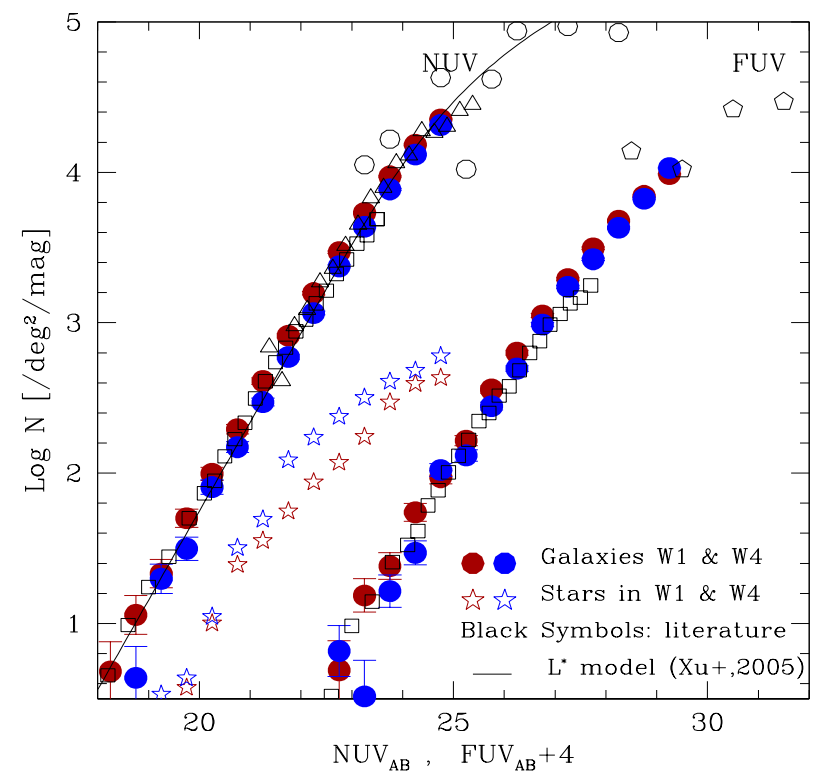

Fig. 8. GALEX number counts in W1 (red symbols) and W4 (blue symbols) regions for the galaxies (filled circles) and the stellar population (open stars). The FUV magnitudes are shifted by +4 mag. Open black symbols are from the literature (triangles: Hoversten et al. 2009; circles and pentagons: Gardner et al. 2000; squares: Xu et al. 2005).

stars and galaxies (see Sect. 3), and they are compared with the literature.

\section{Classification of stars, QSOs, and galaxies}

To minimise the misclassification of stars and galaxies in the final samples, special care must be applied to the star-galaxy separation. For instance in the W4 field, near the Galactic plane, the VVDS survey (based on a pure $i<22.5$ target selection) found more than $32 \%$ of the spectroscopic sources to be stars (Garilli et al. 2008). To distinguish between stars and galaxies without damaging the completeness of our sample, we performed a classification based on three different diagnostics:

- We used the maximum surface brightness versus magnitude (hereafter $\mu_{\max }-m_{\mathrm{obs}}$ ) plane where bright point-like sources are clearly separated from galaxies (see Bardeau et al. 2005; Leauthaud et al. 2007), as shown in Fig. 9. The method relies on the fact that the light distribution of a point-like source and its magnitude are proportional. Moreover, it allows us to detect objects with light distributions that are more peaked than the point-spread function (PSF), which are generally fake detections (gray dots) and then removed. In the inset panel of Fig. 9, one can see that the $\mu_{\max }$ selection (yellow dots), noted $\boldsymbol{S}^{\mu}$, is only relevant up to a certain surface brightness limit, $\mu_{\max }^{\lim }$. The limit depends on the seeing, thus varies from tile to tile and with the considered passband. We performed the $\mu_{\max }$ selection on the $g, r, i$ passbands and imposed that a source is classified as compact if it belongs to $\boldsymbol{S}^{\mu}$ in at least two bands.

- We used the SED fitting technique to compare the reduced $\chi^{2}$ obtained with the galaxy templates described in Sect. 4 and a representative stellar library from Pickles (1998). An object belongs to $S^{\chi}$ if $\chi_{\text {star }}^{2} / \chi_{\text {gal }}^{2}<2$, where the factor 2 has been set to catch $90 \%$ of the point-like objects.

- To minimise the loss of galaxies in the above steps, we finally used the $(g-z)$ vs. $(z-K)$ colour diagram (equivalent to the 


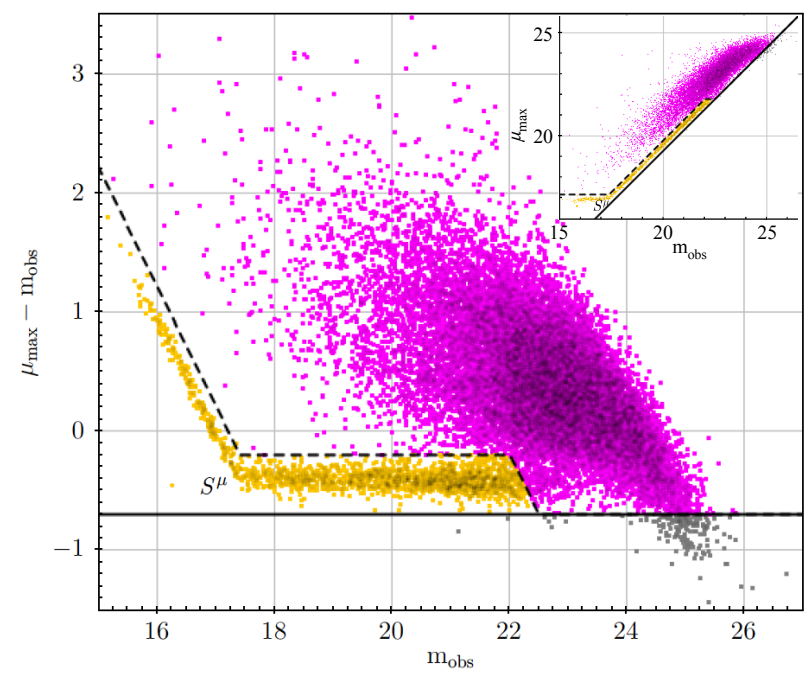

Fig. 9. Photometric classification between point-like and extended sources in the $\mu_{\max }-m_{\text {obs }}$ vs. $m_{\text {obs }}$ plane (for one tile of the CFHTLS in the $i$-band). The dashed line represents our selection of point-like sources $\left(\boldsymbol{S}^{\mu}\right.$, yellow dots). All objects lying below the solid line (gray dots) are considered as false detections.

$B z K$ plane of Daddi et al. 2004) when available to isolate the stellar sequence, noted $S^{B z K}$.

We adopted the latter as a sine qua non condition to be a star, which leads to these final combinations for a source to be classified as star:

$$
\begin{aligned}
{\left[\boldsymbol{S}^{\mu} \cup \boldsymbol{S}^{\chi}\right] \cap \boldsymbol{S}^{B z K} } & \text { if } \mu_{\max }<\mu_{\max }^{\lim } \\
\boldsymbol{S}^{\chi} \cap \boldsymbol{S}^{B z K} & \text { if } \mu_{\max }>\mu_{\max }^{\lim }
\end{aligned}
$$

In the bright point-like source domain $\left(\mu_{\max }<\mu_{\max }^{\text {lim }}\right)$, we define as QSO the objects lying on the galaxy side of the $B z K$ diagram (corresponding to $S^{\mu} \cap ! S^{B z K}$ ). Dominated by their nucleus, the emission of those AGNs is currently poorly linked to their stellar mass. They represent less than $0.5 \%$ of the global sample. By using 1119 spectroscopically confirmed AGNs from the SDSS and VIPERS surveys, we confirmed that our photometric selection of QSOs is able to catch $65 \%$ of them, without losing a single galaxy.

All the objects that were not defined as stars or QSO were then considered as galaxies. We verified on a sample of 1241 spectroscopically confirmed stars that we thereby catch $97 \%$ of them, while we keep more than $99 \%$ of our spectroscopic galaxy sample. With this selection we finally found and removed $\sim 8 \%$ and $\sim 19 \%$ of stars at $K_{\mathrm{s}}<22$ for W1 and W4, respectively (in the unmasked area). The star number counts in the WIRCam and GALEX (FUV, NUV) passbands are shown separately for the W1 and W4 fields in Figs. 3 and 8, respectively. The density of stars in W4 is higher than in W1 at all wavelengths and reaches almost a factor 3 in $K_{\mathrm{s}}$ band.

Finally, in Fig. 10 we show the star and galaxy distributions in the $B z K$ colour diagram for the two fields separately. The lowdensity contours of the galaxy distributions can overlap the stellar locus. Our photometric sample of QSOs (red and blue stars) is also compared with spectroscopically confirmed AGNs (orange dots) from the SDSS and VIPERS surveys.

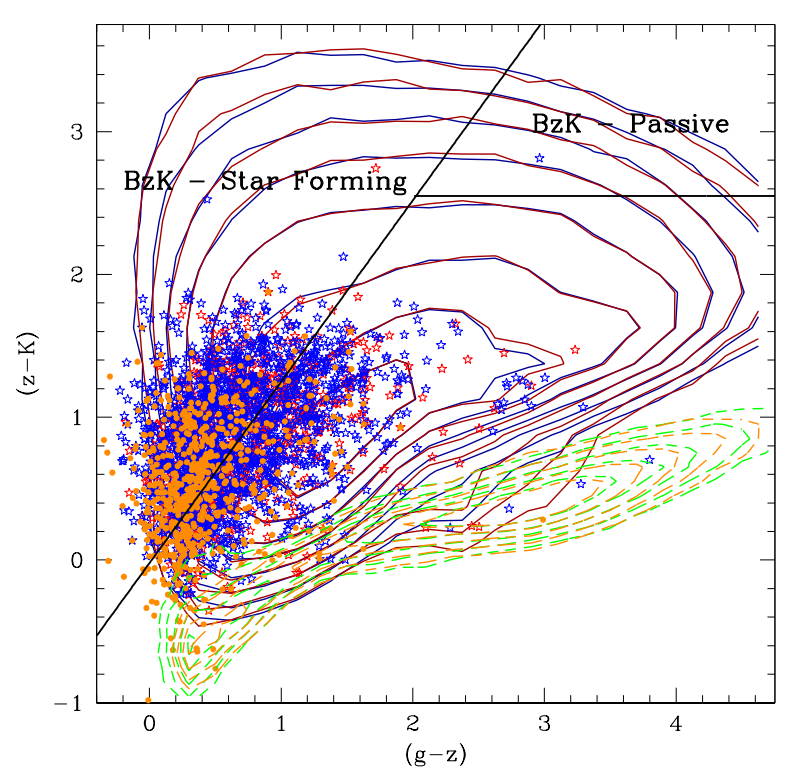

Fig. 10. Distribution of galaxies, stars, and QSOs in the $(z-K)$ vs. $(g-z)$ colour diagram. Density levels are in logarithmic scale $(\Delta=$ 0.3 dex) for galaxies (solid lines in W1 and W4 fields shown blue and red, respectively) and stars (dashed lines in green and orange). Objects from our QSO samples are shown as open stars (blue: W1; red: W4), while the SDSS/VIPERS AGN sample is shown as orange dots. The solid black lines delineate the high-redshift $B z K$ populations (starforming and passive galaxies).

\section{Photometric redshifts}

\subsection{Photometric redshift measurement}

Thanks to the enlarged wavelength coverage, we can estimate new photometric redshifts for the T0007 CFHTLS sources in the VIPERS regions, which also benefit from the large spectroscopic coverage for calibration purposes. The photometric redshifts were computed with the SED-fitting code LE PHARE (Arnouts et al. 2002; Ilbert et al. 2006). We adopted the galaxy SED templates used by Ilbert et al. (2009) (31 empirical templates from Polletta et al. 2007 and 12 star-forming templates from Bruzual and Charlot models, Bruzual \& Charlot 2003). They were modified to better fit the photometry discussed here. We used the large VIPERS spectroscopic redshift sample (see Coupon et al. 2015, for details). The extinction was added as a free parameter with a reddening excess $E(B-V)<0.3$ following different laws: Prevot et al. (1984), Calzetti et al. (2000), plus a dusty Calzetti curve including a bump at $2175 \AA$ A. No extinction was allowed for SEDs redder than Sc. The Prevot et al. (1984) extinction law was allowed for templates redder than SB3 templates (see Ilbert et al. 2009) and Calzetti et al. (2000) for bluer ones. Finally, the systematic disagreement between the photometry and the template library was corrected by LE PHARE according to the method described by Ilbert et al. (2006). In brief, the code tracks a systematic shift between the predicted and the observed magnitudes in each band at known redshifts. Since our observation area is divided into 54 CFHTLS tiles of $\lesssim 1 \mathrm{deg}^{2}$ with photometry varying from tile to tile, we performed a tile-by-tile colour optimisation. We used the median offset over all the tiles when there were not enough available galaxies with spectroscopic redshift in the tile $\left(N_{\mathrm{gal}}^{\mathrm{spec}} \leq 100\right)$, which was the case in 12 tiles. 
Table 2. Characteristics of the spectroscopic redshift sample.

\begin{tabular}{lccc}
\hline \hline $\begin{array}{l}\text { Spectroscopic } \\
\text { survey }\end{array}$ & $N_{\mathrm{gal}}^{\mathrm{spec}}$ & $i_{\text {med }}$ & $\mathcal{F}_{\%} \begin{array}{c}i<\mathbf{2 2 . 5} \\
i>22.5\end{array}$ \\
\hline VIPERS & 33896 & 21.7 & $60.5 \underset{4.3}{\mathbf{9 5 . 7}}$ \\
PRIMUS & 14725 & 21.7 & $26.3_{21.7}^{\mathbf{7 8 . 3}}$ \\
SDSS-BOSS & 4075 & 19.0 & $7.3_{0.0}^{\mathbf{1 0 0 . 0}}$ \\
VVDS Wide & 1630 & 21.6 & $2.9_{3.5}^{\mathbf{9 6 . 5}}$ \\
VVDS Deep & 457 & 23.1 & $0.8_{\mathbf{7 5 . 0}}^{\mathbf{2 5 . 0}}$ \\
UDSz & 1220 & 23.1 & $2.2_{\mathbf{7 2 . 7}}^{\mathbf{2 7 . 3}}$ \\
\hline Total sample & 51396 & 21.6 & $100^{\mathbf{8 9 . 4}}$ \\
\hline
\end{tabular}

Notes. We report the number of spectroscopic redshifts $\left(N_{\text {gal }}^{\mathrm{spec}}\right)$, the median value in the $i$-band $\left(i_{\text {med }}\right)$, the contribution of each survey to the total spectroscopic sample $\left(\mathcal{F}_{\%}\right)$, and their relative fraction of galaxies with $i<22.5$ and $i>22.5$.

\subsection{Comparison with the spectroscopic sample}

Our WIRCam survey has been designed to cover the VIMOS Public Extragalactic Survey (VIPERS; Guzzo et al. 2014) carried out with the VIMOS $^{8}$ spectrograph. We used the first public data release (PDR1) described in Garilli et al. (2014), which provides a $i<22.5$-limited sample of $\sim 57000$ spectroscopic redshifts at $0.5<z<1.2^{9}$ with high precision $\left(\sigma_{v}=\right.$ $140 \mathrm{~km} \mathrm{~s}^{-1}$, which corresponds to $\left.0.0005(1+z)\right)$.

The spectroscopic sample was complemented with other surveys listed in Table 2. We outline their characteristics in this table. We used the VIMOS Very Deep Survey (VVDS, Le Fèvre et al. 2013) "Wide" ( $i<22.5)$ in W4 and "Deep" $(i<24)$ in $\mathrm{W} 1$, spanning the redshift ranges $0.05<z<2$ and $0.05<z<5$ respectively. Only a fraction of the VVDS survey intersects our WIRCam coverage. The deep part of the VVDS survey allowed us to test the photometric redshifts in a fainter regime and at higher redshift. For the same purpose, we also included the $K<23$ limited UKIDSS spectroscopic Ultra Deep Survey (UDSz, Bradshaw et al. 2013; McLure et al. 2013), which has redshifts in $0<z<4.8$ obtained with the VIMOS and FORS2 VLT spectrographs. We used redshifts from the Baryon Oscillation Spectroscopic Survey (BOSS, Dawson et al. 2013) when available. BOSS is a bright-limited $(i<19.9)$ spectroscopic survey from the Sloan Digital Sky Survey (SDSS), providing redshifts up to $z=0.7$ in the entire region of our $K_{\mathrm{s}}$-coverage. Finally, we included the most secure redshifts from the PRIsm MUlti-object Survey (PRIMUS; Coil et al. 2011). This sample is based on low-resolution spectra $(\lambda / \Delta \lambda \sim 40)$ and is limited to $i \sim 23$ (see Cool et al. 2013, for more details).

We selected only the most secure spectroscopic redshifts, which means confidence levels above $95 \%$ for high-resolution surveys:

\section{- flags 3 and 4 with VIMOS (VIPERS, VVDS, UDSz); \\ - flags A and B with FORS2 (UDSz); \\ - $z$-warning 0 in BOSS;}

and $\sigma<0.005$ (8\% of outliers with $\delta z /(1+z)>5 \sigma)$ for PRIMUS flag-4 redshifts. When they were available, the

\footnotetext{
8 Please refer to Le Fèvre et al. (2003) for more details.

9 The targets were preselected in colour space to enhance the probability of observing galaxies at $z>0.5$. This technique results in $\sim 40 \%$ sampling rate with a single pass of VIMOS.
}

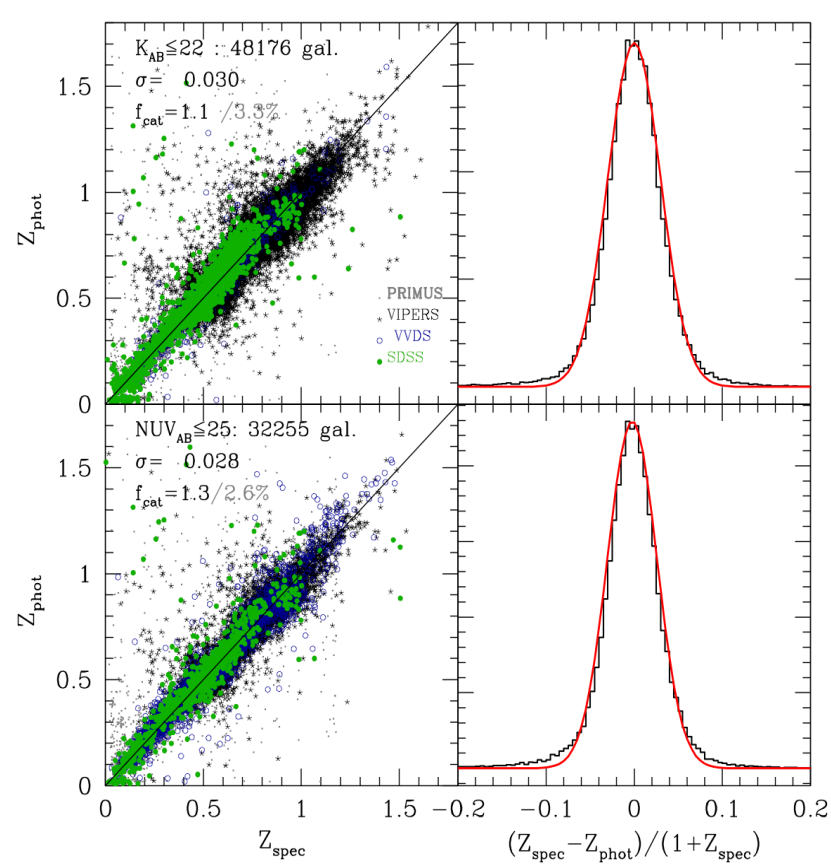

Fig. 11. Comparison of our photometric redshifts with the VVDS, VIPERS, PRIMUS, and BOSS most secure spectroscopic redshifts, for galaxies with $K \leq 22$ (top panels) and NUV $\leq 25$ (bottom panels). We report the dispersion $\left(\sigma_{\mathrm{NMAD}}\right)$ by combining all the surveys, while the catastrophic fractions $\left(f_{\text {cat }}\right.$ defined as $\left.|\Delta z| /\left(1+z_{\mathrm{s}}\right)>0.15\right)$ are given for all surveys but PRIMUS (black value), which is shown separately (gray value). The observed distributions of photometric errors are compared to a Gaussian distribution with the same $\sigma$ (right panels).

redshift measurements from VIPERS were used. Otherwise, the measurements from the deepest high-resolution spectra were favoured.

In total, we collected a sample of $\sim 51396$ high-quality spectroscopic redshifts to calibrate and measure the accuracy of our photometric redshifts over the entire area of the survey.

Figure 11 shows the comparisons between our photometric redshifts and the spectroscopic redshifts for the $K \leq 22$ (top panels) and NUV $\leq 25$ (bottom panels) selected samples. By using the NMAD to define the scatter ${ }^{10}$, we find $\sigma_{\Delta z /(1+z)} \lesssim 0.03$ for both samples with an outlier rate ${ }^{11} \eta \sim 1 \%$. The photometric redshift errors are well described by a Gaussian distribution with the same $\sigma$ for the two samples, as illustrated in the right panels of Fig. 11. As reported in Table 2, we recall that this comparison is dominated by spectroscopic galaxies with $i \leq 22.5$. In Fig. 12 we show the $\sigma$ (left axis, open symbols and solid lines) and the catastrophic fraction rate (right axis, solid symbols and dashed lines) as a function of magnitude ( $i$ : top panel; $K_{\mathrm{s}}$ : middle panel; NUV: bottom panel) for the W1 and W4 fields. As expected, a slight deterioration of the accuracy at $i>22$ is visible.

It is interesting to note that when the PRIMUS redshifts are included in our statistics, we observe a strong increase in the average number of catastrophic failures (by a factor 2,3) in the NUV - and $K_{\mathrm{s}}$-selected samples (as reported in Fig. 11, gray values). We show separately the catastrophic fraction for PRIMUS spectra in Fig. 12 (gray filled triangles). A strong increase is observed toward the faint magnitudes, while we do not observe this behaviour for the high-resolution spectroscopic surveys (SDSS, VVDS, VIPERS, and UDSz). This could suggest that the

${ }^{10} \sigma_{\Delta z /(1+z)}=1.48$ median $\left(\left|z_{\text {spec }}-z_{\text {phot }}\right| /\left(1+z_{\text {spec }}\right)\right)$.
${ }^{11} \eta$ is the percentage of galaxies with $\Delta z /(1+z)>0.15$. 


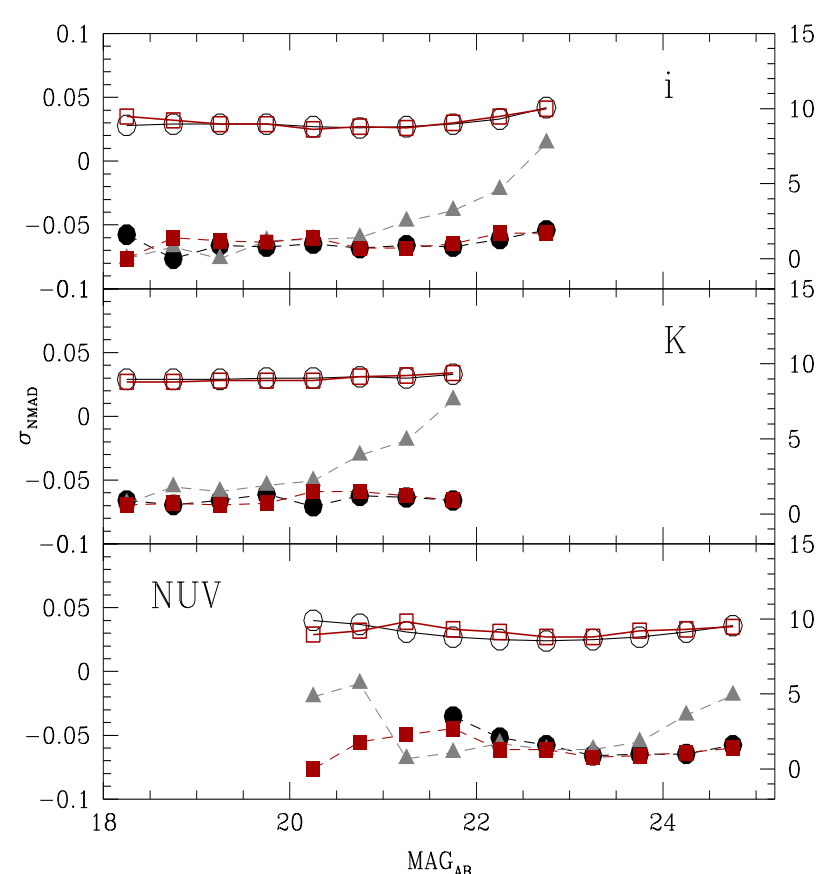

Fig. 12. Comparison of the dispersion $\left(\sigma_{\mathrm{NMAD}}\right)$ as a function of magnitude (top panel: $i$; middle panel: $K_{\mathrm{s}}$; bottom panel: NUV). The $\sigma_{\mathrm{NMAD}}$ for the W1 (black circles) and W4 (red squares) fields correspond to the open symbols and solid lines and refer to the left $y$-axis. The fraction of catastrophic redshifts are reported as field symbols and dashed lines and refer to the right $y$-axis (\%). The PRIMUS spectroscopic sample in $\mathrm{W} 1$ field is shown separately (gray triangles).

PRIMUS redshift flags are too optimistic regarding the quality of the spectra and redshift measurements at fainter magnitudes ${ }^{12}$.

Nevertheless, the large amount of high-resolution spectroscopic redshifts available in our area allows us to reliably quantify the quality of our photometric redshifts. As shown in Fig. 12, the dispersion is always below $\sigma \sim 0.04$ and the catastrophic fraction (excluding PRIMUS) always below $2 \%$ over the entire magnitude range at $i \leq 23$ (both down to $K_{\mathrm{s}} \sim 22$ and NUV 25). However, we recall that values quoted in Figs. 11 and 12 do not account for the selection bias introduced by the spectroscopic sample against blue or red galaxies when moving toward fainter NUV or $K$ magnitudes, respectively.

\subsection{Comparison with the VIDEO photometric redshifts}

Half of the three VISTA pointings from the VIDEO survey (Jarvis et al. 2013) overlaps with our WIRCam observations (see Fig. 2). We can compare the photometric redshifts derived from the deep VIDEO $Y, J, H, K$ data (reaching $K \sim 23.5$ ) with our WIRCam photometric redshifts to partially remove the spectroscopic bias discussed above.

We first matched the full CFHTLS-T0007 optical catalogue with the NIR VIDEO photometry (within a search radius of $\left.0.5^{\prime \prime}\right)$, to which we added the GALEX photometry. We derived the photometric redshifts for this combined catalogue (GALEXCFHTLS-VIDEO) using the same recipe as described above.

In Figs. 13 and 14 we compare our original photometric redshifts (referred to as $z_{\text {wircam }}$ ) with VIDEO photometric redshifts (referred to as $\left.z_{\text {VISTA }}\right)$ for a $K-\left(K_{\text {VISTA }} \leq 23.5\right)$ and NUV- $(N U V \leq 25.5)$ selected sample. In both figures we split

\footnotetext{
${ }^{12}$ We recall that the average redshift success of PRIMUS declines to
} $45 \%$ at $i \sim 22.5$ (see Cool et al. 2013).
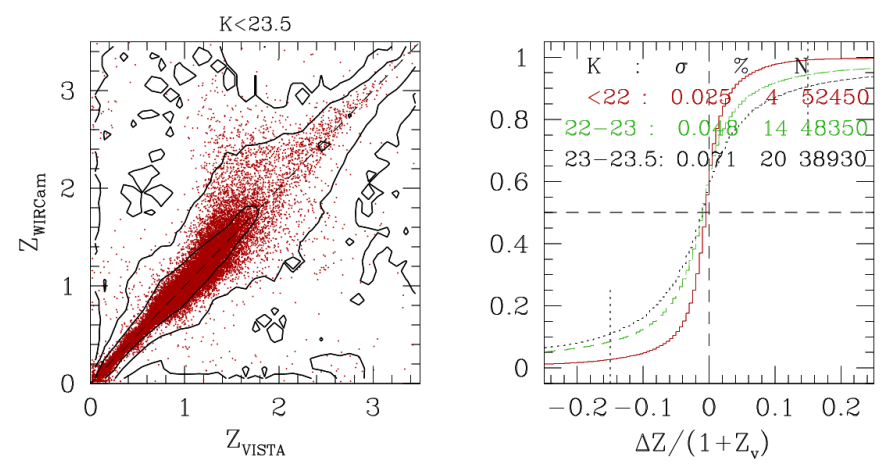

Fig. 13. Comparison of the photometric redshifts measured using either WIRCam $K$ band $\left(z_{\text {wircam }}\right)$ or YJHK photometry from VISTA $\left(z_{\text {VISTA }}\right)$ for galaxies with $K_{\mathrm{VISTA}} \leq 23.5$. The left panel shows the comparison $z_{\text {wircam }}$ vs. $z_{\text {VISTA }}$ for $K_{\text {VISTA }}<22$ (red dots), while the density contours spaced in logarithmic scale of $1 \mathrm{dex}$ are for the faint sample, $22<K_{\mathrm{VISTA}}<23.5$. The right panel shows the cumulative distributions of the difference $\left(\Delta z /\left(1+z_{\mathrm{VISTA}}\right)\right)$ split into three $K$-band subsamples $\left(K_{\mathrm{VISTA}} \leq 22\right.$ : red line; $22<K_{\mathrm{VISTA}}<23$ : green line; $23<K_{\text {VISTA }}<23.5$ : red line). We also report the dispersions $(\sigma)$, the fractions of catastrophic sources (in \%), and the number of sources used.
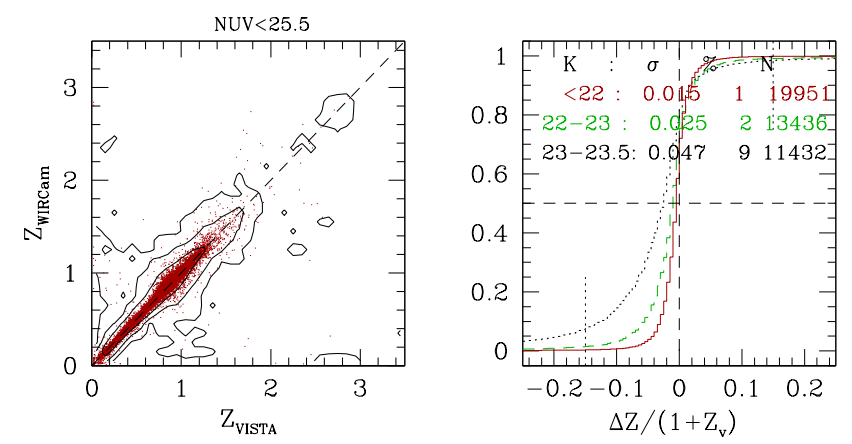

Fig. 14. Same as Fig. 13 for a NUV-selected sample with NUV $\leq 25.5$ and also split into $K_{\mathrm{VISTA}}$ subsamples.

the data into a $K$-bright $\left(K_{\mathrm{VISTA}} \leq 22\right)$ and a $K$-faint $(22 \leq$ $K_{\text {VISTA }} \leq 23.5$ ) sample. In the left panels, we show the oneto-one comparison of the photometric redshifts for the $K$-bright samples (red dots), while the $K$-faint samples are shown with density contours spaced in logarithmic scale of 1 dex (black lines). In the right panels we show the cumulative distributions of the errors $(\Delta z /(1+z))$, and we report the number of sources in each selection, the dispersions, and the catastrophic fractions.

For the $K$-selected sample in Fig. 13, our photometric redshifts are consistent with $z_{\text {VISTA }}(\sigma \sim 0.025, \eta \sim 4 \%)$ up to $z \sim 3$ at bright magnitudes. The $z_{\text {wircam }}$ tend to overestimate the redshifts in between $1<z_{\mathrm{VISTA}}<2$. In the two fainter magnitude bins, the photo- $z$ remains unbiased up to $z \sim 3$, but the dispersions and the catastrophic fractions quickly increase. This is expected since our photometric redshifts are only constrained by the optical magnitudes of the CFHTLS.

For the NUV selected sample in Fig. 14, the photometric redshifts are in excellent agreement up to redshift $z \sim 1.5$. For the bright subsample, $K_{\mathrm{VISTA}} \leq 22$ (red dots), we observe a very small dispersion $(\sigma \sim 0.015)$ and a negligible fraction of outliers. This accuracy holds down to the magnitude range $22 \leq K_{\mathrm{VISTA}} \leq 23$. At fainter magnitudes, $K_{\mathrm{VISTA}} \geq 23$, our photometric redshifts starts to become slightly worse and has a small bias $\Delta z /(1+z) \leq 0.02$. This shows that for our NUVselected samples, we have a good confidence level in the photometric redshifts derived with WIRCam. 


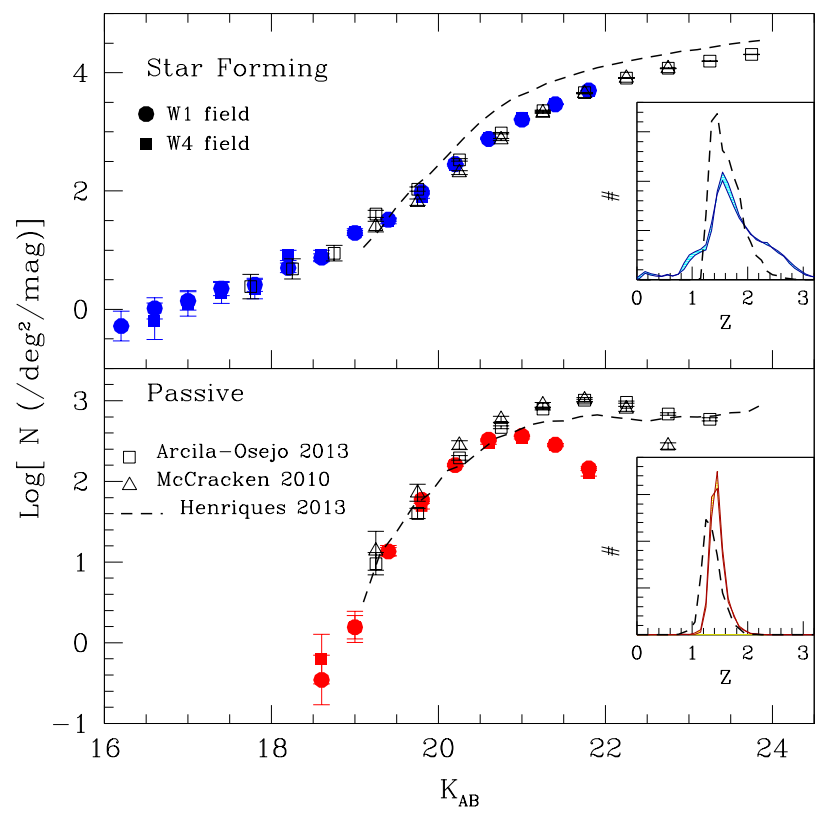

Fig. 15. Differential number counts of star-forming (top) and passive (bottom) $B z K$ galaxies in the $\mathrm{W} 1$ and $\mathrm{W} 4$ fields. We compare with deeper observations in CFHTLS deep fields (open squares, Arcila-Osejo \& Sawicki 2013) and COSMOS (open triangles, McCracken et al. 2010) and model predictions (dashed lines, Henriques et al. 2013) The inset panels show the photometric redshift distributions with $K_{\mathrm{AB}} \leq 22$.

\section{Discussion}

\subsection{BzK selection}

The large area covered by our $K$-band survey and the optical depth of the CFHTLS Wide data makes our sample unique to select a large sample of bright and massive galaxies at high redshift, $z \geq 1.4$, with the $B z K$ colour selection (Daddi et al. 2004). We adopted the criteria from Arcila-Osejo \& Sawicki (2013), who adjusted the selection for the CFHTLS and WIRCam $K$-band photometry. This colour selection is shown in Fig. 10 (solid lines) and provides a sample of $\sim 100000$ star-forming and $\sim 7800$ passive $B z K$ s. The differential number counts are shown in Fig. 15 for the star-forming (top panel) and passive (bottom panel) galaxies down to $K \sim 22$. The error bars are from Poisson statistics. Our results are compared with the deep NIR data in the four CFHTLS deep fields (only the average of the four fields is shown, Arcila-Osejo \& Sawicki 2013) and in the COSMOS field (McCracken et al. 2010). Our results provide robust constraints at the bright end. For the star-forming $B z K s$, we agree well with previous works in the overlapping range and down to our limit, $K_{\mathrm{s}} \sim 22$. For the passive $B z K$ s our result agrees with others at the bright end, but we observe a drop at $K_{\mathrm{s}} \geq 20.7$. Because of the optical gri $\chi^{2}$ image detection, our data suffer from incompleteness against the reddest population. As illustrated in Fig. 5, the 50\% completeness limit corresponds to $\left(z-K_{\mathrm{s}}\right) \sim 3$ at $K_{\mathrm{s}} \sim 21$ and drops to $\left(z-K_{\mathrm{s}}\right) \sim 2.5$ at $K_{\mathrm{s}} \sim 22$ (corresponding to the passive $B z K$ colour threshold). At high redshift, $z \geq 1.4$, our native optical selection introduced a bias against those passive galaxies, which explains the observed drop in density of the passive $B z K$ population at $K_{\mathrm{s}} \geq 20.7$.

In the insets of each panel of Fig. 15, we show the photometric redshift distributions for the two populations in the two fields. The vast majority of $B z K \mathrm{~s}$ is observed at $z_{\text {phot }} \geq 1.2$, as expected from the original work by Daddi et al. (2004), and with a negligible fraction of outliers at low redshift. The star-forming $B z K \mathrm{~S}$ show a large spread in redshift, $1 \leq z \leq 3$, with a peak around $z \sim 1.5$. Passive $B z K$ s also peak at $z \sim 1.5$, but show a narrower distribution $(1.2 \leq z \leq 1.8)$.

We compared the observations with the semi-analytical model of Henriques et al. (2013), based on the Millenium simulations, which has been tuned to reproduce the stellar mass functions at all redshifts. We defined our own colour criteria in the simulations to best distinguish the galaxies with high and low specific SFR (sSFR) at $z \geq 1.4$. We adopted the following criterion: $(z-K)-0.9(g-z) \geq 0.7$ for active and $(z-K)-0.9(g-z) \geq 0.7$ and $(z-K) \geq 2.55$ for passive galaxies. The predicted number densities as a function of magnitude are shown in Fig. 15. The SAM simulation shows similar behaviours as the observed ones for both the star-forming and the passive galaxy populations. The passive population agrees well over the whole magnitude range. For the star-forming population, the simulation over-predicts the density by at least a factor two at magnitudes fainter than $K_{\mathrm{AB}} \sim 20$. This is highly significant in view of the uncertainties in the observations. The redshift distributions in the simulation are concentrated in the redshift range $1 \leq z \leq 2$, and the redshift peaks are at slightly lower redshifts than our $B z K$ photo- $z$ s for both the active and the passive $B z K$ populations. This difference can be partially due to the photo- $z$ bias affecting this redshift range, which are spread toward higher redshift as discussed in Sect. 4.3.

\subsection{Galaxy morphology in the NUVrK diagram}

The star-forming and quiescent galaxies form a bimodal distribution in rest-frame colours or D4000 break. However, the separation between the two populations is not straightforward because of the presence of dust obscured star-forming galaxies when a single colour diagnostic is used. Williams et al. (2010) broke this degeneracy by using a bi-colour $(U-V)$ vs. $(V-J)$ diagram $(U V J)$, which better separates the passive red galaxies from the dusty red galaxies. Arnouts et al. (2013) adopted a similar diagram (NUV $-r)$ vs. $(r-K)$, (NUVrK) to optimize the role of the current SFR and the dust obscuration.

In this section we use the high image quality of the CFHTLS and the large volume to investigate the morphological appearance of well-resolved low- $z$ galaxies according to their location in the NUVrK diagram. The full sample of galaxies with $z \leq 0.25$ was split into seven broad categories shown by different colours in the top left panel of Fig. 16, while the sSFR is colour-coded in the bottom right panel. In the central part of Fig. 16, we show the a set of gri colour stamps (generated with the STIFF software; Bertin 2011) of the largest galaxies (with a major axis $A \geq 50$ pixels). They are randomly placed within subregions (splitting each category into four subcategories).

We are indeed limited in the number of galaxies that we can show in this diagram, and we favoured the most attractive galaxies. However, we generated several realisations of the diagram and found that it does not change the points we address below on the morphological appearances within the NUVrK diagram:

- Star-forming galaxies fall in the categories I to IV. The bluest galaxies (category I-II) have spiral or irregular morphologies and often show clumps of star formation and more disturbed disks. They have high specific SFRs that can reflect a high gas fraction. For the oldest population (redder $(r-K)$ colour, category II-III) we observe a larger portion 


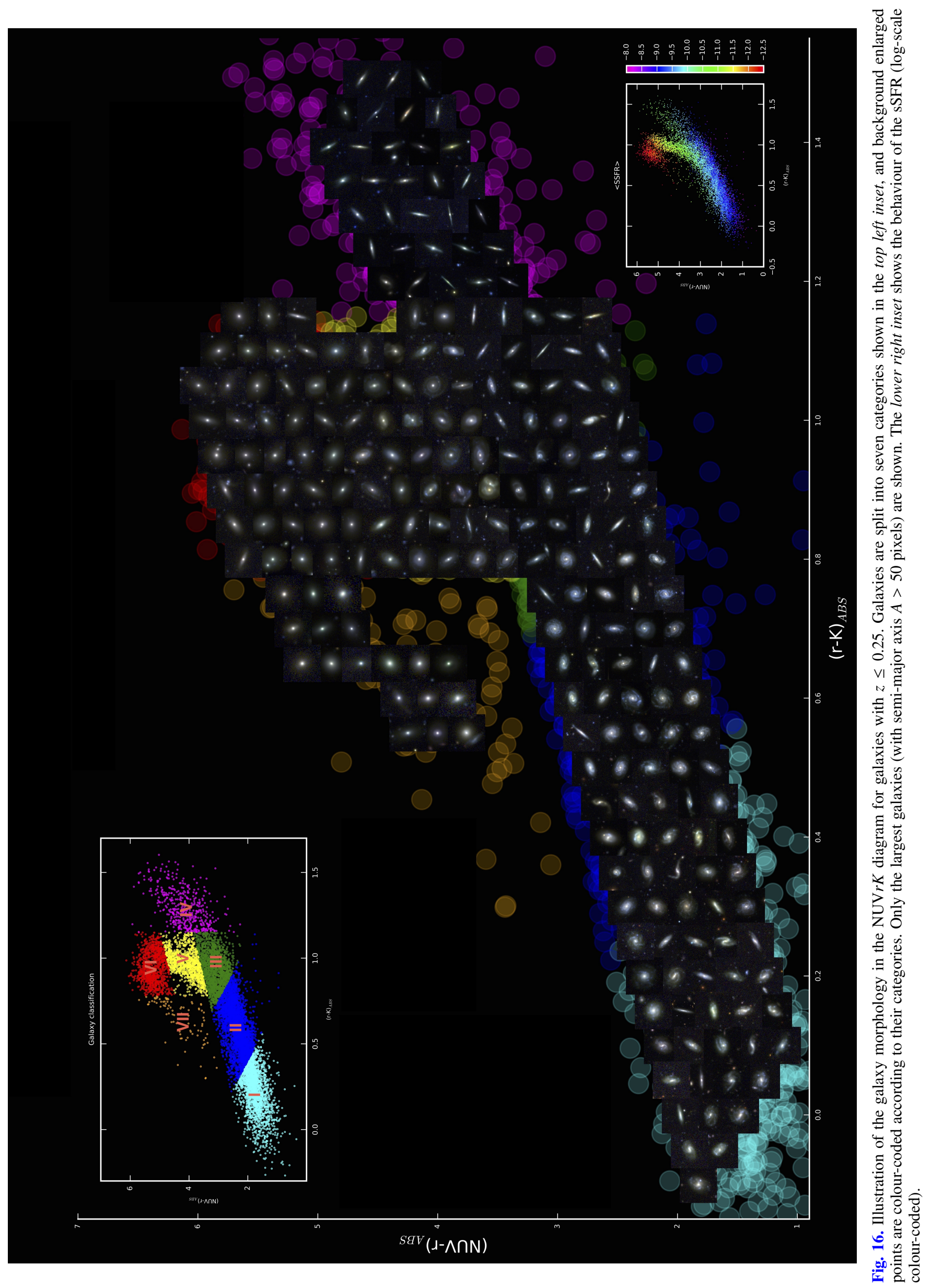


of grand-design spiral galaxies. They are more massive and have a lower sSFR. Their morphologies suggest that these spiral galaxies are more settled and more stable than their lowest mass counterparts, which is consistent with the kinematic analysis of spiral galaxies from Kassin et al. (2012).

- Category IV (purple) reveals a specific population of edgeon galaxies. This confirms the claim of Arnouts et al. (2013) that only highly inclined disk galaxies can produce such extreme infrared excess (IRX) and red $(r-K)$ colour (see also Patel et al. 2011, for a similar conclusion from UVJ diagram).

- Category V (yellow) shows a variety of morphologies. According to their decreasing SSFR, it represents massive galaxies most probably transiting from the star-forming to the quiescent populations. We observe a significant fraction of mergers and post-mergers with extended tidal debris, consistent with the scenario of Hopkins et al. (2006), where mergers trigger both the quenching through AGN feedback and the morphological transformation. Conversely, some of these interaction or merger systems can also be the result of gas accreted onto red galaxies from a minor gas-rich merger, which triggers new star formation activity. This rejuvenation process is observed in the local Universe (Salim \& Rich 2010; Thomas et al. 2010) and also predicted in hydrodynamical simulations (Trayford et al. 2016). We also observe several spiral galaxies with bars and rings within the disks. This could point us toward the importance of secular evolution in the star formation regulation and the disk instability leading to a morphological change. Sheth et al. (2008) showed that most massive spiral galaxies have a higher fraction of bars, associated with a bulge and have redder colours, consistent with this result.

- Category VI (red) is exclusively dominated by massive S0 and elliptical morphologies (e.g., Bell 2008). At $z \sim 1$, Ilbert et al. (2010) observed a significant fraction of quiescent galaxies with disk-dominated morphologies (see also Bundy et al. 2010), but such a population is not observed in our local sample.

- Category VII (orange) shows a small population of galaxies with blue $(r-K)$ colour and (NUV $-r)$ redder than the star-forming categories. In the companion paper (Moutard et al. 2016), we interpret this region through the quenching channel that characterises young (blue $(r-$ $K$ ) colour) low-mass galaxies. Evolutionary tracks in the NUVrK diagram showed that their quenching should be faster than 0.5 Gyr. Environmental quenching such as harassment or ram-pressure stripping can be consistent with this timescale, but we exclusively observe elliptical morphology for this population (with a more compact effective radius than elliptical in category VI). The environmental quenching mechanisms discussed above do not explain the morphological transformation into elliptical. It may in turn favour a rapid morphological transformation by merging for these low-mass galaxies (Schawinski et al. 2014).

As shown in this section, the UV-optical-NIR colours combined with the morphological information provides a good basis for studying star formation activity, evolutionary timescales, and quenching processes of local galaxies. Our description is only qualitative, and we postpone a more detailed analysis of the few hundred galaxies for which detailed morphological information can be measured to a future work (Baillard et al. 2011).

\section{Conclusions}

We presented the multi-wavelength observations collected in the VIPERS region within the $\mathrm{W} 1$ and $\mathrm{W} 4$ fields of the CFHTLS. The NIR observations were adjusted to provide $K_{\mathrm{s}}$-band photometry of the VIPERS spectroscopic sources $\left(i_{\mathrm{AB}} \leq 22.5\right)$. By comparing them with external deep data, we estimated the depth of our WIRCam to be $K_{\mathrm{s}} \sim 22$ at $3 \sigma$. We combined this dataset with GALEX observations. Because of the large GALEX PSF, we developed a specific procedure to derive GALEX photometry in crowded fields based on $u$-band optical priors. We estimated the reliability of the method with simulations and found that we reach robust flux measurement down to NUV $\sim 25$ at $5 \sigma$. With this multi-wavelength catalogue (GALEX, optical, $K_{\mathrm{s}}$ ) we estimated new reliable photometric redshifts for more than one million galaxies at $z \leq 1.5$, with $\sigma_{z} \leq 0.04$ and $\eta \leq 2 \%$ down to $i \sim 23$. We also pushed our analysis at higher redshift with the $B z K$ galaxy populations. Despite our native gri detection method, we showed that we can assemble a complete sample of $B z K$ star-forming galaxies down to $K_{\mathrm{s}} \sim 22$ and $B z K$ passive galaxies down to $K_{\mathrm{s}} \sim 20.7$,

The deep multi-wavelength dataset combined with the unprecedented large area makes this sample perfectly suited for statistical analysis and constrains the rare massive galaxies. In the companion paper (Moutard et al. 2016), we use this sample to determine the massive end of the GSMF for quiescent and starforming galaxies based on photometric redshifts at $0.2 \leq z \leq 1.5$ with high accuracy, while Davidzon et al. (2013) performed a similar analysis in between $0.5 \leq z \leq 1.3$ with the VIPERS spectroscopic sample. The main feature of the VIPERS MultiLambda Survey is its ability to investigate the galaxy properties as a function of environment at high redshift. Davidzon et al. (2016) use the VIPERS spectroscopic redshifts to investigate the changes in the GSMFs in high and low density regions at $z \sim 1$ while Coupon et al. (2015) use photometric redshifts combined with galaxy clustering and galaxy-galaxy lensing to provide new constraints on the relation between galaxies and their host dark matter halos. In a forthcoming work, we will reconstruct the cosmic web from the complete VIPERS spectroscopic sample and investigate how galaxy properties change in dependance on their location within the cosmic web.

We released the photometry of the VIPERS Multi Lambda Survey over its $24 \mathrm{deg}^{2}$. The images, catalogues, and photometric redshifts for $\sim 1.5$ million $[(\mathrm{NUV} \leq 25) \cup(K \leq 22)]$ sources are available online ${ }^{13}$

Acknowledgements. We gratefully thank C. Moreau from CeSAM/LAM for her major contribution to the building of the database. We would like to thank M. Jarvis and B. Haeussler for giving us access to the VIDEO dataset in the XMMLSS field. We also wish to thank R. Overzier for providing us semi-analytical simulations, and L. Arcila-Osejo for helping us with the $B z K$ compilations. This research is in part supported by the Centre National d'Etudes Spatiales (CNES) and the Centre National de la Recherche Scientifique (CNRS) of France, and the ANR Spin(e) project (ANR-13-BS05-0005, http://cosmicorigin.org). L.G. acknowledges support of the European Research Council through the Darklight ERC Advanced Research Grant (\# 291521). This paper is based on observations obtained with MegaPrime/MegaCam, a joint project of CFHT and CEA/DAPNIA, and with WIRCam, a joint project of CFHT, Taiwan, Korea, Canada and France, at the Canada-France-Hawaii Telescope (CFHT).The CFHT is operated by the National Research Council (NRC) of Canada, the Institut National des Sciences de l'Univers of the Centre National de la Recherche Scientifique (CNRS) of France, and the University of Hawaii. This work is based in part on data products produced at TERAPIX available at the Canadian Astronomy Data Centre as part of the Canada-France-Hawaii Telescope Legacy Survey, a collaborative project of NRC and CNRS. We thank the TERAPIX team for the reduction of all the WIRCAM images and the preparation of the catalogues matching

13 http: //cesam. lam. fr/vipers-mls/ 
with the T0007 CFHTLS data release. This paper is based on observations made with the Galaxy Evolution Explorer (GALEX). GALEX is a NASA Small Explorer, whose mission was developed in cooperation with the Centre National d'Études Spatiales (CNES) of France and the Korean Ministry of Science and Technology. GALEX is operated for NASA by the California Institute of Technology under NASA contract NAS5-98034. This paper uses data from the VIMOS Public Extragalactic Redshift Survey (VIPERS). VIPERS has been performed using the ESO Very Large Telescope, under the "Large Programme" 182.A-0886. The participating institutions and funding agencies are listed at http: //vipers.inaf.it. This paper uses data from the VIMOS VLT Deep Survey (VVDS) obtained at the ESO Very Large Telescope under programs 070.A-9007 and 177.A-0837, and made available at the CeSAM data center, Laboratoire d'Astrophysique de Marseille, France. Funding for PRIMUS is provided by NSF (AST-0607701, AST-0908246, AST-0908442, AST-0908354) and NASA (Spitzer-1356708, 08-ADP08-0019, NNX09AC95G). Funding for SDSS-III has been provided by the Alfred P. Sloan Foundation, the Participating Institutions, the National Science Foundation, and the US Department of Energy Office of Science. The Participating Institutions of the SDSS-III Collaboration are listed at http://www.sdss3.org/

\section{References}

Aragón-Calvo, M. A., van de Weygaert, R., \& Jones, B. J. T. 2010, MNRAS, 408,2163

Arcila-Osejo, L., \& Sawicki, M. 2013, MNRAS, 435, 845

Arnouts, S., Moscardini, L., Vanzella, E., et al. 2002, MNRAS, 329, 355

Arnouts, S., Le Floc'h, E., Chevallard, J., et al. 2013, A\&A, 558, A67

Baillard, A., Bertin, E., de Lapparent, V., et al. 2011, A\&A, 532, A74

Bardeau, S., Kneib, J.-P., Czoske, O., et al. 2005, A\&A, 434, 433

Bell, E. F. 2008, ApJ, 682, 355

Bertin, E. 2006, in Astronomical Data Analysis Software and Systems XV, ASP Conf. Ser., 351, 112

Bertin, E. 2011, Astrophysics Source Code Library [record ascl : 1110.006]

Bertin, E., \& Arnouts, S. 1996, A\&AS, 117, 393

Bielby, R., Hudelot, P., McCracken, H. J., et al. 2012, A\&A, 545, A23

Bouché, N., Dekel, A., Genzel, R., et al. 2010, ApJ, 718, 1001

Boulade, O., Charlot, X., Abbon, P., et al. 2000, in SPIE Conf. Ser. 4008, eds. M. Iye, \& A. F. Moorwood, 657

Bradshaw, E. J., Almaini, O., Hartley, W. G., et al. 2013, MNRAS, 433, 194

Bruzual, G., \& Charlot, S. 2003, MNRAS, 344, 1000

Bundy, K., Scarlata, C., Carollo, C. M., et al. 2010, ApJ, 719, 1969

Calzetti, D., Armus, L., Bohlin, R. C., et al. 2000, ApJ, 533, 682

Cattaneo, A., Dekel, A., Devriendt, J., Guiderdoni, B., \& Blaizot, J. 2006, MNRAS, 370, 1651

Chabrier, G. 2003, PASP, 115, 763

Codis, S., Pichon, C., \& Pogosyan, D. 2015, MNRAS, 452, 3369

Coil, A. L., Blanton, M. R., Burles, S. M., et al. 2011, ApJ, 741, 8

Conroy, C. 2013, ARA\&A, 51, 393

Conseil, S., Vibert, D., Amouts, S., et al. 2011, in Astronomical Data Analysis Software and Systems XX, eds. I. N. Evans, A. Accomazzi, D. J. Mink, \& A. H. Rots, ASP Conf. Ser., 442, 107

Cool, R. J., Moustakas, J., Blanton, M. R., et al. 2013, ApJ, 767, 118

Cooper, M. C., Newman, J. A., Weiner, B. J., et al. 2008, MNRAS, 383, 1058

Coupon, J., Arnouts, S., van Waerbeke, L., et al. 2015, MNRAS, 449, 1352

Cucciati, O., Iovino, A., Marinoni, C., et al. 2006, A\&A, 458, 39

Daddi, E., Cimatti, A., Renzini, A., et al. 2004, ApJ, 617, 746

Davidzon, I., Bolzonella, M., Coupon, J., et al. 2013, A\&A, 558, A23

Davidzon, I., Cucciati, O., Bolzonella, M., et al. 2016, A\&A, 586, A23

Dawson, K. S., Schlegel, D. J., Ahn, C. P., et al. 2013, AJ, 145, 10

de la Torre, S., Guzzo, L., Peacock, J. A., et al. 2013, A\&A, 557, A54

Erben, T., Hildebrandt, H., Miller, L., et al. 2013, MNRAS, 433, 2545

Farouki, R., \& Shapiro, S. L. 1981, ApJ, 243, 32

Furusawa, H., Kosugi, G., Akiyama, M., et al. 2008, ApJS, 176, 1

Gardner, J. P., Brown, T. M., \& Ferguson, H. C. 2000, ApJ, 542, L79

Garilli, B., Le Fèvre, O., Guzzo, L., et al. 2008, A\&A, 486, 683

Garilli, B., Guzzo, L., Scodeggio, M., et al. 2014, A\&A, 562, A23

Giavalisco, M., Ferguson, H. C., Koekemoer, A. M., et al. 2004, ApJ, 600, L93

Guillaume, M., Llebaria, A., Aymeric, D., Arnouts, S., \& Milliard, B. 2005, in

Image Processing: Algorithms and Systems, Neural Networks and Machone

Learning, eds. D. Astola, Egiazarian, Nasrabadi, \& Rizvi, Proc. SPIE, 6064, 11

Gunn, J. E., \& Gott, III, J. R. 1972, ApJ, 176, 1
Guzzo, L., Scodeggio, M., Garilli, B., et al. 2014, A\&A, 566, A108 Haines, C. P., Gargiulo, A., \& Merluzzi, P. 2008, MNRAS, 385, 1201 Henriques, B. M. B., White, S. D. M., Thomas, P. A., et al. 2013, MNRAS, 431, 3373

Hildebrandt, H., Erben, T., Kuijken, K., et al. 2012, MNRAS, 421, 2355

Hopkins, P. F., Somerville, R. S., Hernquist, L., et al. 2006, ApJ, 652, 864

Horiuchi, S., Kameno, S., \& Ohishi, M. 2001, in Astronomical data analysis software and systems X, ASP Conf. Ser., 238, 529

Hoversten, E. A., Gronwall, C., Vanden Berk, D. E., et al. 2009, ApJ, 705, 1462 Hudelot, P., Cuillandre, J.-C., Withington, K., et al. 2012, VizieR Online Data Catalog: II/317

Ilbert, O., Arnouts, S., McCracken, H. J., et al. 2006, A\&A, 457, 841

Ilbert, O., Capak, P., Salvato, M., et al. 2009, ApJ, 690, 1236

Ilbert, O., Salvato, M., Le Floc'h, E., et al. 2010, ApJ, 709, 644

Jarvis, M. J., Bonfield, D. G., Bruce, V. A., et al. 2013, MNRAS, 428, 1281

Kassin, S. A., Weiner, B. J., Faber, S. M., et al. 2012, ApJ, 758, 106

Katz, N., Keres, D., Dave, R., \& Weinberg, D. H. 2003, in The IGM/Galaxy Connection. The Distribution of Baryons at $z=0$, eds. J. L. Rosenberg, \& M. E. Putman, Astrophys. Space Sci. Libr., 281, 185

Kauffmann, G., White, S. D. M., Heckman, T. M., et al. 2004, MNRAS, 353, 713

Kereš, D., Katz, N., Weinberg, D. H., \& Davé, R. 2005, MNRAS, 363, 2

Kovač, K., Lilly, S. J., Cucciati, O., et al. 2010, ApJ, 708, 505

Kovač, K., Lilly, S. J., Knobel, C., et al. 2014, MNRAS, 438, 717

Kron, R. G. 1980, ApJS, 43, 305

Larson, R. B., Tinsley, B. M., \& Caldwell, C. N. 1980, ApJ, 237, 692

Lawrence, A., Warren, S. J., Almaini, O., et al. 2007, MNRAS, 379, 1599

Le Fèvre, O., Saisse, M., Mancini, D., et al. 2003, in Instrument Design and Performance for Optical/Infrared Ground-based Telescopes, eds. M. Iye, \& A. F. M. Moorwood, SPIE Conf. Ser., 4841, 1670

Le Fèvre, O., Vettolani, G., Garilli, B., et al. 2005, A\&A, 439, 845

Le Fèvre, O., Cassata, P., Cucciati, O., et al. 2013, A\&A, 559, A14

Leauthaud, A., Massey, R., Kneib, J.-P., et al. 2007, ApJS, 172, 219

Madau, P., \& Dickinson, M. 2014, ARA\&A, 52, 415

Marmo, C., \& Bertin, E. 2008, Astronomical Data Analysis Software and Systems, ASP Conf. Ser., 394, 619

Martin, D. C., Fanson, J., Schiminovich, D., et al. 2005, ApJ, 619, L1

Matsuoka, Y., \& Kawara, K. 2010, MNRAS, 405, 100

McCracken, H. J., Capak, P., Salvato, M., et al. 2010, ApJ, 708, 202

McCracken, H. J., Milvang-Jensen, B., Dunlop, J., et al. 2012, A\&A, 544, A156

McLure, R. J., Pearce, H. J., Dunlop, J. S., et al. 2013, MNRAS, 428, 1088

Morrissey, P., Schiminovich, D., Barlow, T. A., et al. 2005, ApJ, 619, L7

Moutard, T., Arnouts, S., Ilbert, O., et al. 2016, A\&A, 590, A103

Newman, J. A., Cooper, M. C., Davis, M., et al. 2013, ApJS, 208, 5

Oke, J. B. 1974, ApJS, 27, 21

Patel, S. G., Kelson, D. D., Holden, B. P., Franx, M., \& Illingworth, G. D. 2011, ApJ, 735, 53

Peng, Y.-J., Lilly, S., Kovač, K., et al. 2010, ApJ, 721, 193

Pickles, A. J. 1998, PASP, 110, 863

Polletta, M., Tajer, M., Maraschi, L., et al. 2007, ApJ, 663, 81

Prevot, M. L., Lequeux, J., Prevot, L., Maurice, E., \& Rocca-Volmerange, B. 1984, A\&A, 132, 389

Puget, P., Stadler, E., Doyon, R., et al. 2004, in Ground-based Instrumentation for Astronomy, eds. A. F. M. Moorwood, \& M. Iye, SPIE Conf. Ser., 5492, 978

Quadri, R., Marchesini, D., van Dokkum, P., et al. 2007, AJ, 134, 1103

Regnault, N., Conley, A., Guy, J., et al. 2009, A\&A, 506, 999

Salim, S., \& Rich, R. M. 2010, ApJ, 714, L290

Schawinski, K., Urry, C. M., Simmons, B. D., et al. 2014, MNRAS, 440, 889

Scoville, N., Aussel, H., Brusa, M., et al. 2007, ApJS, 172, 1

Scoville, N., Arnouts, S., Aussel, H., et al. 2013, ApJS, 206, 3

Sheth, K., Elmegreen, D. M., Elmegreen, B. G., et al. 2008, ApJ, 675, 1141

Sousbie, T. 2011, MNRAS, 414, 350

Szalay, A. S., Connolly, A. J., \& Szokoly, G. P. 1999, AJ, 117, 68

Tempel, E., Stoica, R. S., \& Saar, E. 2013, MNRAS, 428, 1827

Thomas, D., Maraston, C., Schawinski, K., Sarzi, M., \& Silk, J. 2010, MNRAS, 404, 1775

Trayford, J. W., Theuns, T., Bower, R. G., et al. 2016, MNRAS, submitted [arXiv: 1601.07907]

Walcher, J., Groves, B., Budavári, T., \& Dale, D. 2011, Ap\&SS, 331, 1

Williams, R. J., Quadri, R. F., Franx, M., et al. 2010, ApJ, 713, 738

Wong, K. C., Blanton, M. R., Burles, S. M., et al. 2011, ApJ, 728, 119

Xu, C. K., Donas, J., Arnouts, S., et al. 2005, ApJ, 619, L11

Zehavi, I., Zheng, Z., Weinberg, D. H., et al. 2005, ApJ, 630, 1 